\title{
From SBD to SBH: the elastic-plastic plate
}

\author{
DANILO PERCIVALE ${ }^{\dagger}$ \\ Dipartimento di Metodi e Modelli Matematici, Università di Genova, Piazzale Kennedy, \\ Fiera del Mare, Padiglione D, Genova, Italia \\ AND \\ FRANCO TOMARELLI \\ Dipartimento di Matematica Francesco Brioschi, Politecnico, Piazza Leonardo da Vinci 32, \\ 20133, Milano, Italia
}

[Received 18 October 2000 and in revised form 17 September 2001]

\begin{abstract}
A description of a flat elastic perfectly plastic plate is obtained as a variational limit of a thin elastic 3D body with damage at small scale. A model of a rigid plastic slab is described in a similar way.

Keywords: Free discontinuity problems; $\Gamma$-convergence; bounded deformation; elasticity and damage.
\end{abstract}

\section{Introduction}

The asymptotic behaviour of variational integrals depending on vector-valued functions with free discontinuities may describe damaged thin structures by mean of dimension reduction in the framework of $\Gamma$-convergence.

In this paper we derive a variational model of an elastic-plastic plate with flat unstressed configuration by an asymptotic analysis of a thick elastic body with small mesoscopic cracks. We also study the case of a rigid plastic slab.

The elastic-perfectly plastic behaviour of a plate undergoing small deformations has been studied by coupling bulk energy of elastic type and plastic energy concentrated on a priori unknown yield lines: existence and regularity of minimizers that are Special Bounded Hessian functions (SBH) have been proved in several papers [14-17,43]. A simplified model of such stored energy is given by a functional of the following kind:

$$
\int_{\Sigma}\left|\nabla^{2} w\right|^{2} \mathrm{~d} \mathcal{L}^{2}+\mathcal{H}^{1}\left(S_{D w}\right)+\int_{S_{D w}}|[D w]| \mathrm{d} \mathcal{H}^{1},
$$

where $\Sigma \subset \mathbb{R}^{2}$ is the reference configuration of the plate, $w \in S B H(\Sigma)$ is the scalar-valued transverse displacement, $\nabla^{2} w$ is the absolutely continuous part of $D^{2} w$ and $S_{D w}$ denotes the singular set of $D w, \mathcal{L}$ and $\mathcal{H}$ denote, respectively, the Lebesgue and Hausdorff measure.

The stored energy of elastic bodies with small cracks undergoing small deformations can be described in the frame of Special Bounded Deformation functions (SBD). We introduce here the

†Email: percival@dima.unige.it

"Email: fratom@mate.polimi.it 
following model of the related stored energy:

$$
\int_{U}|\mathcal{E}(\mathbf{v})|^{2} \mathrm{~d} \mathcal{L}^{3}+\mathcal{H}^{2}\left(J_{\mathbf{v}}\right)+\int_{J_{\mathbf{v}}}\left|[\mathbf{v}] \odot v_{\mathbf{v}}\right| \mathrm{d} \mathcal{H}^{2}
$$

where the open set $U \subset \mathbb{R}^{3}$ is the reference configuration of the body, $\mathbf{v}: U \subset \mathbb{R}^{3} \rightarrow \mathbb{R}^{3}$ is a vector field with special bounded deformation (that is to say, v belongs to $S B D(U)$ ), $\mathcal{E}(\mathbf{v})$ is the absolutely continuous part of the linear strain tensor e $(\mathbf{v})=\operatorname{sym} D \mathbf{v}, J_{\mathbf{v}}$ is the jump set of $\mathbf{v}$ (the points $\mathbf{x}$ where $\mathbf{v}$ has two different one-sided Lebesgue limits $\mathbf{v}^{+}(\mathbf{x}), \mathbf{v}^{-}(\mathbf{x})$ with respect to a suitable direction $v_{\mathbf{v}}(\mathbf{x})$ ), while $[\mathbf{v}]=\mathbf{v}^{+}-\mathbf{v}^{-}$and $\odot$ denotes the symmetric tensor product.

The first term in (0.2) represents the elastic energy in undamaged regions, the second one is a surface energy (area of material surfaces where damage occurs [32]), and the third one describes a weak resistance of the material to compression or crack opening and is related to the Barenblatt model of damage [7]. The last term allows us to deal with nontrivial loads, even without artificial confinement of the body.

A stored energy similar to (0.2), but without the third term, has been introduced and studied in $[3,9]$. For a different but related approach to the coupling of damage and strain in one dimension see [10]. On this subject we refer also to [27-29, 45].

Minimization of (0.2) is the linearized version of minimization of the nonlinear elastic energy coupled with a surface energy according to the Barenblatt model of quasi-static formation of cracks $[7,12]$ : note that the linear growth in the third term of $(0.2)$ is essential in the analysis, since sublinearity would prevent coerciveness even in BD frame, while subadditivity is necessary in order to have semi-continuity [11: Proposition 7.1].

In this paper we study a variational relationship between the functionals $(0.1)$ and $(0.2)$ by showing that the elastic perfectly plastic energy $(0.1)$ of the plate is the variational limit (as $\varepsilon \rightarrow 0^{+}$) of the fracture-elastic energy of a three-dimensional body with thickness of order $\varepsilon$ described as follows, where appropriate weights are given to the various terms in (0.2): we set $\Sigma^{\varepsilon}=\Sigma \times(-\varepsilon, \varepsilon)$ and

$$
\frac{3}{2} \int_{\Sigma^{\varepsilon}}|\mathcal{E}(\mathbf{v})|^{2} \mathrm{~d} \mathcal{L}^{3}+\frac{\varepsilon^{2}}{2} \mathcal{H}^{2}\left(J_{\mathbf{v}}\right)+\varepsilon \int_{J_{\mathbf{v}}}\left|[\mathbf{v}] \odot v_{\mathbf{v}}\right| \mathrm{d} \mathcal{H}^{2} .
$$

We prove that minimizers of energy $(0.1)$ are limit of minimizers of $(0.3)$ as the thickness $2 \varepsilon$ goes to zero, and that $\varepsilon^{-3}$ rescaled energy (0.3) converges to (0.1), without a priori assuming any formal asymptotic expansion of (0.3) minimizers. Our proofs are valid for general bulk loads. Here, for simplicity, the analysis is detailed assuming homogeneous Dirichlet boundary conditions.

Additional information about functional (0.3) with small but strictly positive $\varepsilon$ can be obtained by the recovery sequence (5.23) defined in Section 5: the total energy of such recovery sequence energy exhibits an excess from minimality of order $\varepsilon^{3}$.

Concrete slabs undergoing small deformations are sometimes described as thin plates whose elastic deformation turns out to be irrelevant if compared with plastic flow occurring along a priori unknown plastic-yield lines [41]. Such situation can be modelled [17] by coupling rigid transverse deformations with plastic hinges along an unknown pattern of lines, and assuming that on these lines the deformation is continuous but the gradient may have jump discontinuity of rank 1: the stored energy of the slab is then

$$
\mathcal{H}^{1}\left(S_{D w}\right)+\int_{S_{D w}}|[D w]| \mathrm{d} \mathcal{H}^{1}
$$


where $\Sigma \subset \mathbb{R}^{2}$ is the reference configuration of the slab, and $w \in \operatorname{SBH}(\Sigma)$ is the scalar-valued transverse displacement and satisfies the constraint $\nabla^{2} w \equiv 0$ in $\Sigma$, that is to say, $w$ is a piecewise affine function in $\Sigma$.

Here we show that energy (0.4) is the variational limit of an energy of an elastic $2 \varepsilon$-thick elastic board with small cracks, analogous to (0.3) but with a differently weighted first term:

$$
\varepsilon^{-1} \int_{\Sigma^{\varepsilon}}|\mathcal{E}(\mathbf{v})|^{2} \mathrm{~d} \mathcal{L}^{3}+\frac{\varepsilon^{2}}{2} \mathcal{H}^{2}\left(J_{\mathbf{v}}\right)+\varepsilon \int_{J_{\mathbf{v}}}\left|[\mathbf{v}] \odot \nu_{\mathbf{v}}\right| \mathrm{d} \mathcal{H}^{2} .
$$

The main results are Theorem 2.1 (asymptotic behaviour of the constant in Korn-Poincare inequality as the open set shrinks in one single direction), Theorem 3.1 (existence of minima of the approximating problems provided a safe load condition is fulfilled), Theorem 3.4 (variational approximation of the plate energy) and Theorem 3.6 (convergence of minimizers to equilibria of the elastic plastic plate), Theorem 3.8 (safe load condition for equilibrium of an elastic plastic plate) and Theorem 4.2 (variational approximation of the rigid plastic slab). The techniques of this paper rely on $\Gamma$ convergence methods [21,23].

The coupling of bulk energy and surface energy causes the main difficulty in the analysis since none of energies $(0.1)-(0.5)$ is convex. We cannot use a straightforward scaling of the Korn-Poincaré inequality, since a dilation of the plate thickness does not map the space of rigid displacements into itself: to overcome this difficulty, we show explicitly the asymptotic behaviour of the constant appearing in the inequality, as the plate thickness goes to 0 .

Moreover, we never consider thickness averages (which could jeopardize the functional frame of allowed damage, since the projection of vertical cuts with finite total area may have infinite length), but we study the approximating functionals in a fixed re-scaled domain (blow-up of the thin plate and slab).

We emphasize that all the results are proved without assuming the Kirchhoff cinematic restriction $\mathrm{e}(\mathbf{v}) \cdot \mathbf{n}=\mathbf{0}$ (see $[33,39]$; obviously such restriction can be skipped in the purely elastic case too, see [1]). In the last section we show some analogies and differences in the analysis if Kirchhoff assumption is made: Theorem 6.2 and Remark 6.3 clarify the gap between the two frameworks.

Actually, by assuming Kirchhoff cinematic restriction, we are able to deduce a quantitative estimate on the amount of damages (see Theorem 6.4). Such estimate may be interpreted as an analytical deduction of the fact that cracks compatible with equilibrium have a smaller size than the Griffith critical length [32].

The one-dimensional problem (approximation of an elastic plastic beam via micro-cracked thin rod) has been studied in [37] (flat beam with Kirchhoff hypothesis) and [38] (curved beam with Kirchhoff hypothesis). Boundary value and obstacle problems with energy (0.2) are studied in [20].

In the elastic context (both for plates and beams) and the Sobolev spaces framework (without allowing fractures in the approximating problems, and hence without plasticity in the limit problem) a statement analogous to Theorems 3.4, 3.6 was proved by [18] (see also [19]).

The purely plastic frame for plates was studied in [36]. The approximation of membrane and thin films (obtained by different weights in (0.3)) is dealt with in [12,13,31].

Regularity for essential minimizers of energy (0.1) with prescribed transverse load and Neumann boundary condition were proven in [16] under smallness condition of the load in $L^{q}(\Omega)$ with $q>2$ : 
in such case energy (0.1) for a minimizer can be written as

$$
\int_{\Sigma \backslash \overline{S_{D w}}}\left|D^{2} w\right|^{2} \mathrm{~d} \mathcal{L}^{2}+\mathcal{H}^{1}\left(\overline{S_{D w}}\right)+\int_{\overline{S_{D w}}}|[D w]| \mathrm{d} \mathcal{H}^{1}
$$

with $w \in C^{0}(\Sigma) \cap C^{2}\left(\Sigma \backslash \overline{S_{D w}}\right)$ and $\left.\mathcal{H}^{1}\left(\left(\overline{S_{D w}} \cap \Sigma\right) \backslash S_{D w}\right)\right)=0$ : that is, closing $S_{D w}$ in $\Sigma$ does not increase length.

Regularity for minimizers of energy (0.4) with prescribed transverse load and Neumann boundary condition were proven in [17] under smallness condition of the load in $L^{q}(\Omega)$ with $q>2$ : in such case energy $(0.4)$ for a minimizer can be written as

$$
\mathcal{H}^{1}\left(\overline{S_{D w}}\right)+\int_{\overline{S_{D w}}}|[D w]| \mathrm{d} \mathcal{H}^{1}
$$

where $w \in C^{0}(\Sigma)$ is affine in each connected component of $\Sigma \backslash \overline{S_{D w}}$ and $\left.\mathcal{H}^{1}\left(\left(\overline{S_{D w}} \cap \Sigma\right) \backslash S_{D w}\right)\right)=$ 0 .

The paper has the following sections:

(1) Functional framework

(2) Asymptotic behaviour of the best constant in the Korn-Poincaré inequality for an $n$ dimensional cylinder whose height tends to null

(3) Approximation of the linear elastic plastic plate (LP)

(4) Approximation of the rigid plastic slab (RS)

(5) Proofs of convergence results

(6) Some remarks about the Kirchhoff cinematic restriction

\section{Functional framework}

We denote by $U$ an open bounded subset of $\mathbb{R}^{n}$ with Lipschitz boundary; by $\mathcal{L}^{n}$ the $n$-dimensional Lebesgue measure and by $\left\{\mathbf{e}_{i}\right\}$ the canonical basis of $\mathbb{R}^{n}$. For a given set $Q \subset \mathbb{R}^{n}$ we denote by $\partial Q$ its topological boundary, by $\mathcal{H}^{m}(Q)$ its $m$-dimensional Hausdorff measure and by $|Q|$ its Lebesgue outer measure; $p^{\prime}=p /(p-1)$ denotes the conjugate exponent of any $p \in[1,+\infty]$. We denote by $B_{\rho}(\mathbf{x})$ the open ball $\left\{\mathbf{y} \in \mathbb{R}^{n} ;|\mathbf{y}-\mathbf{x}|<\rho\right\}$, and we set $B_{\rho}=B_{\rho}(\mathbf{0})$. Moreover, $s \wedge t=\min \{s, t\}$, $s \vee t=\max \{s, t\}$ for every $s, t \in \mathbb{R}$. spt denotes the support of a distribution.

$$
f_{Q} v \mathrm{~d} x=|Q|^{-1} \int_{Q} v \mathrm{~d} x \quad \forall \mathcal{L} \text {-measurable set } Q, \text { and } \mathcal{L} \text {-integrable function } v \text { in } Q .
$$

$M_{k, n}$ denotes the $k \times n$ matrices and $I_{k}$ the identity matrix in $M_{k, k}$; given vectors $\mathbf{a}=\left\{\mathbf{a}_{i}\right\}, \mathbf{b}=\left\{\mathbf{b}_{i}\right\}$, and matrices $A=\left\{A_{i j}\right\}, B=\left\{B_{i j}\right\}$, we set $\mathbf{a} \cdot \mathbf{b}=\sum_{i} \mathbf{a}_{i} \mathbf{b}_{i},(\mathbf{a} \otimes \mathbf{b})_{i j}=\mathbf{a}_{i} \mathbf{b}_{j},(\mathbf{a} \odot \mathbf{b})_{i j}=$ $1 / 2\left(\mathbf{a}_{i} \mathbf{b}_{j}+\mathbf{a}_{j} \mathbf{b}_{i}\right),(A \cdot \mathbf{b})_{i}=\sum_{j} A_{i j} \mathbf{b}_{j},(\mathbf{b} \cdot A)_{j}=\sum_{i} A_{i j} \mathbf{b}_{i},(A B)_{i j}=\sum_{k} A_{i k} B_{k j}, A: B=$ $\sum_{i j} A_{i j} B_{i j},|a|^{2}=a \cdot a,|A|^{2}=A: A=\sup \left\{\sum_{i j} A_{i j} B_{i j}: \sum_{i j} B_{i j}^{2}=1\right\}$.

We say that a subset $E$ of $\mathbb{R}^{n}$ is countably $\left(\mathcal{H}^{n-1}, n-1\right)$ rectifiable if (up to a set of vanishing $\mathcal{H}^{n-1}$ measure) it is the countable union of $C^{1}$ images of bounded subsets of $\mathbb{R}^{n-1}$; if in addition $\mathcal{H}^{n-1}(E)<+\infty$ then we say that $E$ is $\left(\mathcal{H}^{n-1}, n-1\right)$ rectifiable.

For $p \in[1,+\infty]$, and $Y$ a finite-dimensional space, we denote by $L^{p}(U, Y)$ and by $W^{1, p}(U, Y)$ the Lebesgue and Sobolev spaces of functions with values in $Y$, endowed with the usual norms $\|\cdot\|_{L^{p}}$ 
and $\|\cdot\|_{W^{1, p}}$ respectively. $\mathcal{M}(U, Y)$ denotes the space of the bounded measures on $\Omega$ with values in $Y$.

For brevity, we write $L^{p}(U), W^{1, p}(U), \mathcal{M}(U)$ when $Y=\mathbf{R}$.

$|\cdot|_{\mathcal{M}}$ denotes the total variation of a measure in $\mathcal{M}(U, Y)$, i.e.

$$
|\mu|_{\mathcal{M}}=\int_{U} \mathrm{~d}|\mu|=\sup \left\{\int_{U} \sum_{i j} \varphi_{i j} \mathrm{~d} \mu_{i j}: \varphi_{i j} \in C_{0}^{0}(U), \sum_{i j} \varphi_{i j}^{2} \leqslant 1 \text { in } U\right\} .
$$

$\mu^{a}=\frac{\mathrm{d} \mu}{\mathrm{d} \mathcal{L}^{m}}$ is the absolutely continuous part of $\mu$ with respect to $\mathcal{L}^{m}$ and $\mu^{s}=\mu-\mu^{a}$ is the singular part.

If $O \subset U$ is any open set, then $|\mu|_{\mathcal{M}(O)}$ is defined in the same way, with $\varphi_{i j} \in C_{0}^{0}(O)$, and we define a Borel measure $|\mu|$ by setting for every Borel set $B \subset U$

$$
|\mu|(B)=\inf \left\{\int_{O}|\mu| ; B \subset O, O \text { open }\right\} .
$$

Let $\mathbf{v}: U \rightarrow \mathbb{R}^{k}$ be a Borel function, (we write $v$ in the scalar case, $k=1$ ); for $\mathbf{x} \in U$ and $z \in \mathbb{R}^{k}=\mathbb{R}^{k} \cup\{\infty\}$ (the one-point compactification of $\mathbb{R}^{k}$ ), we say that $z$ is the approximate limit of $\mathbf{v}$ at $\mathbf{x}$, and we write

$$
\mathbf{z}=\text { ap } \lim _{\mathbf{y} \rightarrow \mathbf{x}} \mathbf{v}(\mathbf{y}), \quad \text { if, for every } g \in C^{0}\left(\widetilde{\mathbb{R}^{\mathbf{k}}}\right), \quad g(\mathbf{z})=\lim _{\rho \rightarrow 0} \frac{\int_{B_{\rho}(\mathbf{x})} g(\mathbf{v}(\mathbf{y})) \mathrm{d} \mathbf{y}}{\left|B_{\rho}\right|} .
$$

The singular set

$$
S_{\mathbf{v}}:=\left\{\mathbf{x} \in U: \text { ap } \lim _{\mathbf{y} \rightarrow \mathbf{x}} \mathbf{v}(\mathbf{y}) \quad \text { does not exist }\right\}
$$

is a Borel set; for brevity we denote by $\widetilde{\mathbf{v}}: \Omega \backslash S_{\mathbf{v}} \rightarrow \widetilde{\mathbf{R}}^{k}$ the function

$$
\widetilde{\mathbf{v}}(\mathbf{x})=\operatorname{ap} \lim _{\mathbf{y} \rightarrow \mathbf{x}} \mathbf{v}(\mathbf{y}) \text {. }
$$

Let $\mathbf{x} \in U \backslash S_{\mathbf{v}}$ s.t. $\widetilde{\mathbf{v}}(\mathbf{x}) \in \mathbb{R}^{k}$ : we say that $\mathbf{v}$ is approximately differentiable at $\mathbf{x}$ iff there is a $k \times n$ matrix $\nabla \mathbf{v}(\mathbf{x})$ s.t.

$$
\text { ap } \lim _{\mathbf{y} \rightarrow \mathbf{x}} \frac{|\mathbf{v}(\mathbf{y})-\widetilde{\mathbf{v}}(\mathbf{x})-\nabla \mathbf{v}(\mathbf{x})(\mathbf{y}-\mathbf{x})|}{|\mathbf{y}-\mathbf{x}|}=0 .
$$

If $\mathbf{v}$ is a smooth function then $\nabla \mathbf{v}$ coincides with the classical gradient.

We recall the definition of the space of functions with bounded variation in $U$ with values in $\mathbb{R}^{k}$ :

$$
\begin{gathered}
B V\left(U, \mathbb{R}^{k}\right)=\left\{\mathbf{v} \in L^{1}\left(\Omega, \mathbb{R}^{k}\right): D \mathbf{v} \in \mathcal{M}\left(U, M_{k, n}\right)\right\} \\
\|\mathbf{v}\|_{B V U}=\|\mathbf{v}\|_{L^{1}(U)}+\int_{U}|D \mathbf{v}|
\end{gathered}
$$

where $D \mathbf{v}=\left\{D_{j} \mathbf{v}_{i}\right\}_{\substack{i=1, \ldots, k \\ j=1, \ldots m}}$ denotes the distributional derivatives of $\mathbf{v}$.

In the one-dimensional case $(n=1)$ we shall use the notation $\dot{\mathbf{v}}$ in place of $\nabla \mathbf{v}$ and $\mathbf{v}^{\prime}$ instead of $D v$. To simplify notation we set, for any $n \geqslant 1$,

$$
\mathbf{v}, i:=\frac{\partial \mathbf{v}}{\partial x_{i}}=D_{i} \mathbf{v}=D \mathbf{v} \cdot \mathbf{e}_{i} \quad \nabla_{i} \mathbf{v}:=\left(\mathbf{e}_{\mathbf{i}} \cdot \nabla\right) \mathbf{v} \quad i=1, \ldots, n .
$$

For every $\mathbf{v} \in B V\left(U \mathbb{R}^{k}\right)$ the following properties hold: 
(BV1) $\widetilde{\mathbf{v}}(\mathbf{x}) \in \mathbb{R}^{k}$ for $\mathcal{H}^{n-1}$ almost all $\mathbf{x} \in U \backslash S_{\mathbf{v}}$ (see [46: 5.9.6]);

(BV2) $S_{\mathrm{v}}$ has null Lebesgue measure and is countably $\left(\mathcal{H}^{n-1} ; n-1\right)$ rectifiable (see [46: 5.9.6]);

(BV3) $\nabla \mathbf{v}$ exists a.e. in $U$ and coincides with the Radon-Nikodym derivative of $D \mathbf{v}$ with respect to the Lebesgue measure (see [30: 4.5.9(26)]);

(BV4) for $\mathcal{H}^{n-1}$ almost all $\mathbf{x} \in S_{\mathbf{v}}$ there exist $v=v_{\mathbf{v}}(\mathbf{x}) \in \partial B_{1}, \mathbf{v}^{+}(\mathbf{x}), \mathbf{v}^{-}(\mathbf{x}) \in \mathbb{R}^{k}$ (outer and inner trace, respectively, of $\mathbf{v}$ at $\mathbf{x}$ in the direction $v$ ) such that (see [46: 5.14.3] and [30: 4.5.9(15)])

$$
\begin{array}{r}
\lim _{\varrho \rightarrow 0^{+}} \varrho^{-n} \int_{\left\{\mathbf{y} \in B_{\ell}(\mathbf{x}) ;(\mathbf{y}-\mathbf{x}) \cdot v>0\right\}}\left|\mathbf{v}(\mathbf{y})-\mathbf{v}^{+}(\mathbf{x})\right| \mathrm{d} \mathbf{y}=0, \\
\lim _{\varrho \rightarrow 0^{+}} \varrho^{-n} \int_{\left\{\mathbf{y} \in B_{\ell}(\mathbf{x}) ;(\mathbf{y}-\mathbf{x}) \cdot v<0\right\}}\left|\mathbf{v}(\mathbf{y})-\mathbf{v}^{-}(\mathbf{x})\right| \mathrm{d} \mathbf{y}=0, \\
|D v|_{\mathcal{M}} \geqslant \int_{U}\|\nabla v(\mathbf{x})\| \mathrm{d} \mathbf{x}+\int_{S_{v}}\left|\mathbf{v}^{+}(\mathbf{x})-\mathbf{v}^{-}(\mathbf{x})\right| \mathrm{d} \mathcal{H}^{n-1}(\mathbf{x}) ;
\end{array}
$$

(BV5) by setting $j_{\mathbf{v}}=\left(\mathbf{v}^{+}-\mathbf{v}^{-}\right) \otimes v_{\mathbf{v}} \mathrm{d} \mathcal{H}^{n-1}\left\llcorner S_{\mathbf{v}}, C_{\mathbf{v}}=(D v)^{s}-j_{\mathbf{v}}\right.$, we have the decomposition

$$
D \mathbf{v}=\nabla \mathbf{v} \mathrm{d} \mathbf{x}+j_{\mathbf{v}}+C_{\mathbf{v}} .
$$

The space of vector fields with bounded deformation has been introduced to deal with variational problems in perfect plasticity (see $[42,44])$ :

$$
\begin{gathered}
B D(U)=\left\{\mathbf{v} \in L^{1}\left(\Omega, \mathbb{R}^{n}\right): \mathrm{e}(\mathbf{v}):=\frac{1}{2}\left(D \mathbf{v}+(D \mathbf{v})^{T}\right) \in \mathcal{M}\left(U, M_{n, n}\right)\right\} \\
\|\mathbf{v}\|_{B D(U)}=\|\mathbf{v}\|_{L^{1}(U)}+\int_{U}|\mathrm{e}(\mathbf{v})| .
\end{gathered}
$$

$B D(U)$ is the dual of a separable Banach space. For any $\mathbf{v} \in B D(U)$ we define

$$
J_{\mathbf{v}}:=\left\{\mathbf{x} \in S_{\mathbf{v}}: \exists \mathbf{v}^{+}(\mathbf{x}), \mathbf{v}^{-}(\mathbf{x}) \in \mathbb{R}^{n}, v_{\mathbf{v}}(\mathbf{x}) \in \partial B_{1}(0), \text { s.t. (1.1), (1.2) hold with } k=n\right\}
$$

which is the subset of the singular set where $\mathbf{v}$ has one-sided approximate limits with respect to a suitable direction $v_{\mathbf{v}}$ 'normal' to $J_{\mathbf{v}}$. $J_{\mathbf{v}}$ is called the jump set of $\mathbf{v}$ and plays a role analogous to the singular set $S_{\mathrm{v}}$ in the theory of $B V$ functions (see [3]).

We notice that for $\mathbf{v} \in B V\left(U, \mathbb{R}^{k}\right)$, the set $S_{\mathbf{v}} \backslash J_{\mathbf{v}}$ is $\mathcal{H}^{n-1}$ negligible, while it is not known whether the same property holds in $B D(U)$. Moreover, for every $\mathbf{v} \in B D$

(BD1) the linear strain tensor e(v) has the following decomposition:

$$
\mathrm{e}(\mathbf{v})=\mathrm{e}^{a}(\mathbf{v})+\mathrm{e}^{s}(\mathbf{v})=\mathcal{E}(\mathbf{v}) \mathrm{d} \mathbf{x}+\mathrm{e}^{j}(\mathbf{v})+\mathrm{e}^{c}(\mathbf{v})
$$

where $\mathrm{e}^{a}(\mathbf{v})=\mathcal{E}(\mathbf{v}) \mathrm{d} \mathbf{x}$ and $\mathrm{e}^{s}(\mathbf{v})$ are respectively the absolutely continuous and the singular part of e(v) with respect to $\mathcal{L}^{n} ; \mathrm{e}^{j}(\mathbf{v}), \mathrm{e}^{c}(\mathbf{v})$ are respectively the restriction of $\mathrm{e}^{s}(\mathbf{v})$ to $J_{\mathbf{v}}$ and the restriction of $\mathrm{e}^{s}$ to its complement (say the jump and Cantor part of e(v)).

Throughout the paper we denote by $\operatorname{div} \mathbf{v}=\operatorname{Tr} \mathcal{E}(\mathbf{v})$ the absolutely continuous part of the distributional divergence of $\mathbf{v}$.

(BD2) $\mathcal{E}(\mathbf{v})$ can be interpreted as an approximate symmetric differential ([3: Theorem 4.3]):

$$
\lim _{\varrho \rightarrow 0^{+}} \frac{1}{\varrho^{n}} \int_{B_{\varrho}(\mathbf{x})} \frac{|(\mathbf{v}(\mathbf{y})-\mathbf{v}(\mathbf{x})-\mathcal{E}(\mathbf{v}) \cdot(\mathbf{y}-\mathbf{x})) \cdot(\mathbf{y}-\mathbf{x})|}{|\mathbf{y}-\mathbf{x}|^{2}} \mathrm{~d} \mathbf{y}=0 \quad \mathcal{L}^{n} \text { a.e. } x \in U .
$$


(BD3) $J_{\mathbf{V}}$ is a Borel set with null Lebesgue measure and is countably $\left(\mathcal{H}^{n-1}, n-1\right)$ rectifiable (see [3: Proposition 3.5]), and there are $v_{\mathbf{v}}=v_{\mathbf{v}}(x) \in \partial B_{1}, \mathbf{v}^{+}(x), \mathbf{v}^{-}(x)$ (respectively geometric measure theory normal, outer and inner trace in the $v$ direction) $\mathcal{H}^{n-1}$ a.e. in $J_{\mathbf{v}}$, s.t.

$$
\mathrm{e}^{j}(\mathbf{v})=\left(\mathbf{v}^{+}-\mathbf{v}^{-}\right) \odot v_{\mathbf{v}} \mathcal{H}^{n-1}\left\llcorner J_{\mathbf{v}},\right.
$$

and the jump part $\mathrm{e}^{j}(\mathbf{v})$ can be represented on every Borel set $B$ by the formula

$$
\mathrm{e}^{j}(\mathbf{v})(B)=\int_{B \cap J_{\mathbf{v}}}[\mathbf{v}] \odot v_{\mathbf{v}} \mathrm{d} \mathcal{H}^{n-1}, \quad \text { where }[\mathbf{v}]:=\mathbf{v}^{+}-\mathbf{v}^{-} .
$$

(BD4) If $\mathcal{R}$ denotes the set of rigid displacements (the affine maps of type $A \cdot x+b$ where $A \in M_{n, n}$ is skew-symmetric and $b \in \mathbb{R}^{n}$ ), then ([42: Proposition 2.2, 2.3 p. 155] and [3: Theorem 3.1]) for every bounded connected open set $U$ with Lipschitz boundary, and every continuous linear map $R: B D(U) \rightarrow \mathcal{R}$ which leaves fixed the elements of $\mathcal{R}$, there is a constant $c_{1}=c_{1}(U, R)$ such that

$$
\|\mathbf{v}-R(\mathbf{v})\|_{L^{n /(n-1)}(U)} \leqslant c_{1}(U, R)|\mathrm{e}(\mathbf{v})|(U) \quad \forall \mathbf{v} \in B D(U) .
$$

(BD5) If $\psi$ is a continuous semi-norm on $B D(U)$ and a norm on $\mathcal{R}$, then $\psi(v)+\int_{U}|\mathrm{e}(\mathbf{v})|$ is a norm on $B D(U)$ equivalent to $\|\cdot\|_{B D(U)}$. In particular

(BD6) (Korn-Poincaré inequality) If $U$ is the unit cube $(-1,1)^{n}$ and $\partial_{l} U$ denotes its lateral boundary $\left\{\mathbf{x} \in \partial U: \mathbf{x}_{n} \neq \pm 1\right\}$, then there is a constant $c_{2}=c_{2}(U)$ such that

$$
\|\mathbf{v}\|_{L^{n /(n-1)}(U)} \leqslant c_{2}(U)\left(|\mathrm{e}(\mathbf{v})|(U)+\int_{\partial_{l} U}|\mathbf{v}| \mathrm{d} \mathcal{H}^{n-1}\right) \quad \forall \mathbf{v} \in B D(U) .
$$

The constants in BD4, BD6 are invariant by dilations $(\varepsilon U, \varepsilon>0)$ of the cube.

$$
B D(U) \subset L^{s}(U) \quad \forall s \in[1, n /(n-1)] \quad \text { with compact embedding if } s<n /(n-1) .
$$

In the study of elastic-perfectly plastic plates the spaces $B H(\Omega)$ (functions with bounded Hessian) and $\operatorname{SBH}(\Omega)$ have been introduced and studied in [14, 26, 40];

$$
\begin{gathered}
B H(U)=\left\{v \in W^{1,1}(U): D^{2} v \in \mathcal{M}\left(U, M_{n, n}\right)\right\}=\left\{v \in L^{1}(\Omega): D v \in B V\left(U, \mathbb{R}^{m}\right)\right\}, \\
\|v\|_{B H(U)}=\|v\|_{L^{1}(U)}+\|D v\|_{L^{1}(U)}+\left|D^{2} v\right|_{\mathcal{M}} .
\end{gathered}
$$

$B H(U)$ endowed with this norm is the dual of a Banach space.

Now we recall the definition and main properties of the following spaces: functions with special bounded variation (see [25]), vector fields with special bounded deformation (see [3]) and functions with special bounded hessian (see [14]), and point out some of their properties. These spaces are characterized by the property that some combinations of distributional derivatives are special measures in the sense of De Giorgi ([24]). We set

$$
\begin{gathered}
S B V\left(U, \mathbb{R}^{k}\right)=\left\{\mathbf{v} \in B V\left(U, \mathbb{R}^{k}\right): C_{\mathbf{v}} \equiv 0\right\}, \\
S B D(U)=\left\{\mathbf{v} \in B D(U): \mathrm{e}^{c}(\mathbf{v}) \equiv 0\right\}, \\
S B H(U)=\left\{w \in W^{1,1}(U): D w \in S B V\left(U, \mathbb{R}^{n}\right)\right\}, \\
S B V^{2}(U)=\left\{w \in S B V(U): \nabla w \in S B V\left(U, \mathbb{R}^{n}\right)\right\} .
\end{gathered}
$$


We note that $\mathbf{v} \in \operatorname{SBV}\left(U, \mathbb{R}^{k}\right)$ if and only if

$$
\mathbf{v} \in B V\left(U, \mathbb{R}^{k}\right) \quad \text { and } \quad D \mathbf{v}=\nabla \mathbf{v} \mathrm{d} \mathbf{x}+\left(\mathbf{v}^{+}-\mathbf{v}^{-}\right) \otimes v_{\mathbf{v}} \mathrm{d} \mathcal{H}^{n-1}\left\llcorner S_{\mathbf{v}}\right.
$$

where $\mathcal{H}^{n-1}\left\llcorner S_{\mathbf{v}}(B)=\mathcal{H}^{n-1}\left(B \cap S_{\mathbf{v}}\right)\right.$ for any Borel set $B$ (see [2]). Moreover (by [9: Appendix])

$$
\begin{gathered}
S B D(U) \cap B V\left(U, \mathbb{R}^{n}\right)=S B V\left(U, \mathbb{R}^{n}\right), \\
S B V\left(U, \mathbb{R}^{n}\right) \underset{\neq}{\subset} S B D(U) \underset{\neq}{\subset} B D(U) .
\end{gathered}
$$

We remark that $D w=\nabla w$ in $S B H(U)$ and in $B H(U)$, but $D w \neq \nabla w$ in $S B V^{2}(U)$. We set

$$
S_{D w}=\bigcup_{i=1}^{n} S_{D_{i} w} \quad \forall w \in S B H(U), \quad S_{\nabla w}=\bigcup_{i=1}^{n} S_{\nabla_{i} w} \quad \forall w \in S B V^{2}(U),
$$

hence

$$
S_{D w}=S_{\nabla w}, \quad \Delta^{a} w=\nabla \cdot D w, \quad \forall w \in S B H(U) .
$$

By definition $S B H(U)$ is a closed subspace of $B H(U)$ with respect to the strong norm, while it is not closed with respect to the $w^{*}-B H(U)$ topology. In addition we have (see $\left.[14,15]\right)$ :

(SBH1) $\int_{U}\left|D^{2} w\right|=\int_{U}\left|\nabla^{2} w\right| \mathrm{d} \mathbf{x}+\int_{S_{D w}}|[D w]| \mathrm{d} \mathcal{H}^{n-1} \quad$ where $[D v]=(D v)^{+}-(D v)^{-}$.

(SBH2) (embeddings) Let $U \subset \mathbb{R}^{n}(n>1)$ be a bounded open set with the exterior cone property. Then

$$
B H(U) \subset W^{1, q}(U)
$$

with continuous embedding if $q \leqslant \frac{n}{n-1}$; compact embedding if $q<\frac{n}{n-1}$, hence

$$
B H(\Omega) \subset L^{S}(\Omega)
$$

for $s \leqslant \frac{n}{n-2}$ (compactly when the inequality is strict) if $n>2$;

for any $s \geqslant 1$ (compactly for finite $s$ ) if $n=2$.

(SBH3) Let $U \subset \mathbb{R}^{2}$ be a an open set. If $w \in B H(U)$ has compact support in $U$, then

$$
\|w\|_{L^{\infty}(U)} \leqslant \frac{1}{4} \int_{U}\left|D^{2} w\right| .
$$

(SBH4) If a bounded connected Lipschitz open set $U \subset \mathbb{R}^{2}$ has boundary $\partial U$ which is a union of finitely many $C^{2}$ regular arcs, then

$$
B H(U) \subset C^{0}(\bar{U}) .
$$

(SBH5) (Traces) Let $U \subset \mathbb{R}^{n}$ have the same property as in SBH4. Then, two bounded linear maps exist:

$$
\gamma_{0}: B H(\Omega) \rightarrow W^{1,1}(\partial \Omega), \quad \gamma_{1}: B H(\Omega) \rightarrow L^{1}(\partial \Omega)
$$

such that

$$
\gamma_{0}(v)=\left.v\right|_{\partial \Omega}, \quad \gamma_{1}(v)=\left.\frac{\partial v}{\partial N}\right|_{\partial \Omega}
$$

for every $v \in C^{2}(\bar{\Omega})$, where $N$ is the outward normal to $\partial \Omega$. Moreover $\gamma_{1}$ is onto. 
(SBH6) Let $U \subset \mathbb{R}^{n}$ be an open set and $w \in \operatorname{SBH}(U)$. Then

(a) $\left(D^{2} w\right)^{s}=[D w] \otimes v \mathrm{~d} \mathcal{H}^{n-1}\left\llcorner S_{D w}=\left[\frac{\partial w}{\partial v}\right] v \otimes v \mathrm{~d} \mathcal{H}^{n-1}\left\llcorner S_{D w}\right.\right.$,

(b) $\int_{U}\left|\left(D^{2} w\right)^{s}\right|=\int_{S_{D w}}|[D w]| \mathrm{d} \mathcal{H}^{n-1}=\int_{S_{D w}}\left|\left[\frac{\partial w}{\partial v}\right]\right| \mathrm{d} \mathcal{H}^{n-1}$,

(c) $\int_{U}\left|\left(D^{2} w\right)^{s}\right|=\int_{U}\left|\Delta^{s} w\right|$,

where $\frac{\partial w}{\partial v}=v \cdot D w$, and $\left(D^{2} w\right)^{s}$ and $\Delta^{s} w$ denote respectively the singular part of the distributional Hessian and Laplacian of $v$ with respect to $\mathcal{L}^{n}$.

We notice explicitly that $C^{\infty}(\Omega)$ is neither dense in $B H(\Omega)$ nor in $\operatorname{SBH}(\Omega)$ with respect to the strong topology. Nevertheless, if $\Omega$ is strongly Lipschitz, the density holds true with respect to the intermediate topology associated to the distance

$$
d_{2}(u, v)=\|u-v\|_{L^{1}(\Omega)}+\left|\int_{\Omega}\right| D^{2} u\left|-\int_{\Omega}\right| D^{2} v|| .
$$

\section{Asymptotic behaviour of the best constant in the Korn-Poincaré inequality for an $n$ - dimensional cylinder whose height tends to null}

We describe the asymptotic behaviour, when $\varepsilon$ goes to zero, of the constant in a Korn-Poincare inequality (of type (BD6)) for a cylinder $\Omega^{\varepsilon}$ (approximating the plate $\Omega$ ), and in general for an $\varepsilon$-fattened open subset of $\mathbb{R}^{n-1}$, by first estimating from above the blow-up rate of such constant and then by exhibiting a simple example which shows exactly such rate. The body is supposed to be fixed at the lateral boundary: this fact is analytically imposed by a support restriction of admissible deformations, which are defined in a set $\Sigma^{\varepsilon}$ bigger than the reference configuration $\Omega^{\varepsilon}$. The following analysis includes Theorem 4.1 in [37] about flat beams.

THEOREM 2.1 For $n \geqslant 2$ let

$$
\Omega \subset \mathbb{R}^{n-1} \text { be a non-empty bounded connected Lipschitz open set, }
$$

$$
\bar{\Omega} \subset(-L, L)^{n-1} \stackrel{\text { def }}{=} \Sigma
$$

and set

$\Gamma=\partial \Omega, \quad \Omega^{\varepsilon}=\Omega \times(-\varepsilon, \varepsilon), \quad \Sigma^{\varepsilon}=\Sigma \times(-\varepsilon, \varepsilon), \quad \Gamma^{\varepsilon}=\partial \Omega \times(-\varepsilon, \varepsilon) \quad \forall \varepsilon \in(0,1]$.

Then there exists a constant $C_{\Omega}=C(\Omega, L, n)>0$ independent of $\varepsilon$ such that

$$
\begin{gathered}
\|\mathbf{v}\|_{L^{n /(n-1)}\left(\Omega^{\varepsilon}\right)} \leqslant C_{\Omega^{\varepsilon}} \varepsilon^{-1-1 / n}|\mathrm{e}(\mathbf{v})|\left(\Sigma^{\varepsilon}\right) \leqslant C_{\Omega^{2}} \varepsilon^{1-1 / n}\left(|\mathrm{e}(\mathbf{v})|\left(\Omega^{\varepsilon}\right)+\int_{\Gamma^{\varepsilon}}|\mathbf{v}| \mathrm{d} \mathcal{H}^{n-1}\right) \\
\forall \varepsilon \in(0,1] \text { and } \forall \mathbf{v} \in B D\left(\Sigma^{\varepsilon}\right) \text { s.t. } \operatorname{spt} \mathbf{v} \subset \overline{\Omega^{\varepsilon}} . \\
\|\mathbf{v}\|_{L^{s}\left(\Omega^{\varepsilon}\right)} \leqslant(2|\Omega|)^{\frac{1}{s}+\frac{1}{n}-1} C_{\Omega} \varepsilon^{\frac{1}{s}-2}\left(|\mathrm{e}(\mathbf{v})|\left(\Omega^{\varepsilon}\right)+\int_{\Gamma^{\varepsilon}}|\mathbf{v}| \mathrm{d} \mathcal{H}^{n-1}\right) \\
\forall \varepsilon \in(0,1], \forall s \in\left[1, \frac{n}{n-1}\right] \text { and } \forall \mathbf{v} \in B D\left(\Sigma^{\varepsilon}\right) \text { s.t. } \operatorname{spt} \mathbf{v} \subset \overline{\Omega^{\varepsilon}}
\end{gathered}
$$


Before proving Theorem 2.1, we recall some known results and state some preliminary lemmas. Let $Q=(-1,1)^{n}$ be the open cube of $\mathbf{R}^{n}, \varepsilon>0$. By [42: Theorem 2.1 p. 148, Proposition 2.3, and Remark 2.5 p. 155-156], it is easy to see that here is a positive constant $c$, depending only on $n$, such that, for every $\mathbf{v} \in B D(\varepsilon Q)$,

$$
\begin{gathered}
\int_{\partial Q}|\mathbf{v}| \mathrm{d} \mathcal{H}^{n-1} \leqslant c\left(|\mathrm{e}(\mathbf{v})|(Q)+\int_{Q}|\mathbf{v}| \mathrm{d} \mathcal{L}^{n}\right) \\
\left(\int_{Q}|\mathbf{v}|^{\frac{n}{n-1}} \mathrm{~d} \mathcal{L}^{n}\right)^{\frac{n-1}{n}} \leqslant c\left(|\mathrm{e}(\mathbf{v})|(Q)+\int_{\partial_{l} Q}|\mathbf{v}| \mathrm{d} \mathcal{H}^{n-1}\right) \\
\exists \mathbf{w} \in \mathcal{R}(\varepsilon Q): \quad\left(\int_{Q}|\mathbf{v}-\mathbf{w}|^{s} \mathrm{~d} \mathcal{L}^{n}\right)^{1 / s} \leqslant c\|\mathrm{e}(\mathbf{v})\|(\varepsilon Q) \quad \forall s \in\left[1, \frac{n}{n-1}\right] .
\end{gathered}
$$

By scaling $\left(x_{\varepsilon}=\varepsilon x, \mathcal{H}^{n-1}(\mathbf{x})=\mathcal{H}^{n-1}\left(\mathbf{x}_{\varepsilon}\right) / \varepsilon^{n-1}, \mathcal{L}^{n}(\mathbf{x})=\mathcal{L}^{n}\left(\mathbf{x}_{\varepsilon}\right) / \varepsilon^{n}\right)$, we get for all $\mathbf{v} \in B D(\varepsilon Q)$

$$
\begin{gathered}
\int_{\partial(\varepsilon Q)}|\mathbf{v}| \mathrm{d} \mathcal{H}^{n-1} \leqslant c\left(|\mathrm{e}(\mathbf{v})|(\varepsilon Q)+\frac{1}{\varepsilon} \int_{\varepsilon Q}|\mathbf{v}| \mathrm{d} \mathcal{L}^{n}\right) \\
\left(\int_{\varepsilon Q}|\mathbf{v}|^{\frac{n}{n-1}} \mathrm{~d} \mathcal{L}^{n}\right)^{\frac{n-1}{n}} \leqslant c\left(|\mathrm{e}(\mathbf{v})|(\varepsilon Q)+\int_{\partial_{l}(\varepsilon Q)}|\mathbf{v}| \mathrm{d} \mathcal{H}^{n-1}\right) \\
\exists \mathbf{w} \in \mathcal{R}(\varepsilon Q): \quad\left(\int_{\varepsilon Q}|\mathbf{v}-\mathbf{w}|^{s} \mathrm{~d} \mathcal{L}^{n}\right)^{1 / s} \leqslant c \varepsilon^{1-\frac{n}{s^{\prime}}}|\mathrm{e}(\mathbf{v})|(\varepsilon Q) \quad \forall s \in\left[1, \frac{n}{n-1}\right] .
\end{gathered}
$$

LEMMA 2.2 For every $\mathbf{a}, \mathbf{b} \in \mathbb{R}^{n}$ the following inequality holds:

$$
\int_{-\varepsilon}^{\varepsilon}|\mathbf{a}+\mathbf{b} z| \mathrm{d} z \geqslant \tau|\mathbf{b}| \varepsilon^{2}+2(1-\tau)|\mathbf{a}| \varepsilon \quad \forall \varepsilon \geqslant 0, \forall \tau \in[0,1]
$$

Moreover, if $\mathbf{b}=\mathbf{0}$, then equality in (2.9) holds iff $\tau=0$; if $\mathbf{b} \neq \mathbf{0}$, then equality in (2.9) holds iff $\mathbf{a}=\mathbf{0}$ and $\tau=1$.

Proof. The case when $\mathbf{a}=\mathbf{0}$ or $\mathbf{b}=\mathbf{0}$ is trivial.

If $\mathbf{a} \neq \mathbf{0} \neq \mathbf{b}$ then, by convexity of the Euclidean norm we have

$$
|\mathbf{a}+\mathbf{b} z| \geqslant|\mathbf{b} z|+\mathbf{a} \cdot \operatorname{sign}(\mathbf{b} z) \forall z \neq 0, \quad|\mathbf{a}+\mathbf{b} z| \geqslant|\mathbf{a}|+\mathbf{b} \cdot \operatorname{sign}(\mathbf{a}) z
$$

where sign denotes the vector-valued sign function: $\operatorname{sign}(\mathbf{a})=\mathbf{a} /|\mathbf{a}|$. By integration

$$
\int_{-\varepsilon}^{\varepsilon}|\mathbf{a}+\mathbf{b} z| \mathrm{d} z \geqslant \int_{-\varepsilon}^{\varepsilon}|\mathbf{b} z| \mathrm{d} z+\int_{-\varepsilon}^{\varepsilon} \frac{\mathbf{b} z}{|\mathbf{b}||z|} \cdot \mathbf{a} \mathrm{d} z=\varepsilon^{2}|\mathbf{b}|
$$

and

$$
\int_{-\varepsilon}^{\varepsilon}|\mathbf{a}+\mathbf{b} z| \mathrm{d} z \geqslant \int_{-\varepsilon}^{\varepsilon}|\mathbf{a}| \mathrm{d} z+\int_{-\varepsilon}^{\varepsilon} \frac{\mathbf{a}}{|\mathbf{a}|} \cdot \mathbf{b} z \mathrm{~d} z=2 \varepsilon|\mathbf{a}|
$$

and (2.9) is a convex combination of the above inequalities.

If $\mathbf{b}=\mathbf{0}$ the statement about equality is trivial. 
A necessary condition for both equalities in (2.10) is that $\mathbf{a}, \mathbf{b}$ are linearly dependent, say if $\mathbf{b} \neq 0$, that $\mathbf{a}=\lambda \mathbf{b}$ for some $\lambda \in \mathbb{R}$, but in this case

$$
\int_{-\varepsilon}^{\varepsilon}|\mathbf{a}+\mathbf{b} z| \mathrm{d} z=|\mathbf{b}| \int_{-\varepsilon+\lambda}^{\varepsilon+\lambda}|s| \mathrm{d} s=|\mathbf{b}| \psi(\lambda)
$$

hence $\psi^{\prime}(\lambda)=0$, iff $\lambda=0$ and $\psi^{\prime \prime}(\lambda)=2$ for $|\lambda| \leqslant \varepsilon$. Therefore $\int_{-\varepsilon}^{\varepsilon}|\mathbf{a}+\mathbf{b} z| \mathrm{d} z$ achieves its strict absolute minimum value $|\mathbf{b}| \varepsilon^{2}$ at $\lambda=0$ and this proves the last part of the thesis.

Lemma 2.3 For every $\mathbf{a}, \mathbf{b} \in \mathbf{R}^{n}$, we have

$$
|\mathbf{a}||\mathbf{b}| / \sqrt{2} \leqslant|\mathbf{a} \odot \mathbf{b}| \leqslant|\mathbf{a}||\mathbf{b}|
$$

and the first inequality is strict iff $\mathbf{a} \cdot \mathbf{b} \neq 0$, the second is strict iff $|\mathbf{a} \cdot \mathbf{b}|=|\mathbf{a}||\mathbf{b}|$.

Proof. For every $\mathbf{c}, \mathbf{d} \in \mathbb{R}^{n}:|\mathbf{c}|=|\mathbf{d}|=1$, we have

$$
|\mathbf{c} \odot \mathbf{d}|^{2}=\frac{1}{4} \sum_{i} \sum_{j}\left(c_{i}^{2} d_{j}^{2}+c_{j}^{2} d_{i}^{2}+2 c_{i} d_{j} c_{j} d_{i}\right)=\frac{1}{4} \sum_{i}\left(c_{i}^{2}+d_{i}^{2}+2 c_{i} d_{i} \mathbf{c} \cdot \mathbf{d}\right)=\frac{1}{2}+\frac{(\mathbf{c} \cdot \mathbf{d})^{2}}{2}
$$

hence

$$
0<2^{-1 / 2}=\min \{|\mathbf{c} \odot \mathbf{d}|:|\mathbf{c}|=|\mathbf{d}|=1\} \leqslant \frac{|\mathbf{a} \odot \mathbf{b}|}{|\mathbf{a}||\mathbf{b}|} \leqslant 1 \quad \forall \mathbf{a}, \mathbf{b} \in \mathbb{R}^{n} \backslash\{\mathbf{0}\} .
$$

In the following we denote respectively by $\mathbf{x}=\left(x_{1}, \ldots, x_{n-1}\right), z$ and $\mathbf{X}=(\mathbf{x}, z)$ the variables in $\Omega,(-\varepsilon, \varepsilon)$ and $\Omega^{\varepsilon}$, and we show an estimate for piecewise rigid displacements on $\Omega^{\varepsilon}$, related to a finite partition of $\Sigma^{\varepsilon}$ in small cubes: we consider for every $\varepsilon>0$ a finite family $Q_{k}^{\varepsilon}$ of pair wise disjoint open cubes of size $2 \varepsilon$ with edges parallel to coordinate axes and centred in $\mathbf{X}_{k}=\left(\mathbf{x}_{k}, 0\right)$ so that

$$
\Sigma^{\varepsilon}=\operatorname{int} \bigcup_{k} \overline{Q_{k}^{\varepsilon}}
$$

We fix $\varepsilon$ and we drop the index $\varepsilon$ whenever there is no risk of confusion.

We denote by $\partial_{l} Q_{k}$ the lateral boundary of $Q_{k}$, that is the portion of $\partial Q_{k}$ outside the planes $\{z= \pm \varepsilon\}$, by $\mathbf{v}^{+}, \mathbf{v}^{-}$the interior and exterior traces of $\mathbf{v}$ on $\partial_{l} Q_{k}$, by $\mathcal{R}\left(Q_{k}\right)$ the space of rigid displacements in $Q_{k}$ and by $\mathbf{1}_{E}(\mathbf{y})=1$ if $\mathbf{y} \in E, \mathbf{1}_{E}(\mathbf{y})=0$ else, the usual characteristic function of a set E.

LEMmA 2.4 There is $c_{\Omega}=c(\Omega, L, n)>0$ such that for every $\mathbf{w}=\sum_{k} \mathbf{w}_{k} \mathbf{1}_{Q_{k}}$, with spt $\mathbf{w}$ disjoint from $\partial \Sigma \times(-\varepsilon, \varepsilon)$ and $\mathbf{w}_{k}$ in $\mathcal{R}\left(Q_{k}\right) \forall k$, the following estimate holds:

$$
\|\mathbf{w}\|_{L^{n /(n-1)}\left(\Omega^{\varepsilon}\right)} \leqslant c_{\Omega} \varepsilon^{-1-1 / n}|\mathrm{e}(\mathbf{w})|\left(\Sigma^{\varepsilon}\right) \quad \forall \varepsilon \text { s.t. } 0<\varepsilon \leqslant 1 .
$$

Proof. We denote by $\mathbf{x}, z,(\mathbf{x}, z)$ respectively the variables on $\Sigma,(-\varepsilon, \varepsilon)$ and $\Sigma^{\varepsilon}$.

In the following we denote by the same letter $c$ suitable constants which may change in different inequalities, but are independent of $\varepsilon$.

Since $\mathbf{w}$ is a rigid displacement in each small cube $Q_{k}$, there are $A_{k} \in \mathcal{M}^{n, n}$ skew-symmetric matrices and $\mathbf{m}_{k} \in \mathbf{R}^{n}$ such that

$$
\mathbf{w}_{k}(\mathbf{x}, z)=A_{k} \cdot\left(\mathbf{x}-\mathbf{x}_{k}, z\right)+\mathbf{m}_{k} \quad \forall(\mathbf{x}, z) \in Q_{k}, \forall k .
$$


We denote by $\bar{A}_{k} \in \mathcal{M}^{n-1, n-1}$ the skew-symmetric matrix obtained from $A_{k}$ by dropping the $n$th row and the $n$th column ( $A_{k}$ is equal to $\overline{A_{k}}$ trimmed by $\mathbf{b}_{k},-\mathbf{b}_{k}^{T}$ and 0 ), then we get

$$
\mathbf{w}_{k}=\left(\bar{A}_{k} \cdot\left(\mathbf{x}-\mathbf{x}_{k}\right)+z \mathbf{b}_{k},-\mathbf{b}_{k} \cdot\left(\mathbf{x}-\mathbf{x}_{k}\right)\right)+\mathbf{m}_{k} \quad \text { in } Q_{k}
$$

In each $Q_{k}$ we set $\mathbf{w}(\mathbf{x})=\left(\mathbf{a}(\mathbf{x})+z \mathbf{b}(x),-\mathbf{b}(\mathbf{x}) \cdot\left(\mathbf{x}-\mathbf{x}_{k}\right)\right)+\mathbf{m}(\mathbf{x})$ where $\mathbf{a}(\mathbf{x})=\bar{A}_{k} \cdot\left(\mathbf{x}-\mathbf{x}_{k}\right)$, $\mathbf{m}(\mathbf{x})=\mathbf{m}_{k}, \mathbf{b}(\mathbf{x})=\mathbf{b}_{k} \in \mathbb{R}^{n-1}$. We set also $\mathbf{B}(\mathbf{x})=-\sum_{k} \mathbf{b}_{k}\left(\mathbf{x}-\mathbf{x}_{k}\right) \mathbf{1}_{Q_{k}}$.

Hence $\mathbf{a}, \mathbf{b} \in S B V\left(\Sigma, \mathbb{R}^{n-1}\right), \mathbf{m} \in S B V\left(\Sigma, \mathbb{R}^{n}\right), \mathbf{b}$ is constant on each $Q_{k}, \nabla_{\mathbf{x}}(\mathbf{b}) \equiv 0$ in $\Sigma^{\varepsilon}$, $\nabla_{\mathbf{x}}(\mathbf{m}) \equiv 0$ in $\Sigma^{\varepsilon}, \mathcal{E}_{\mathbf{x}}(\mathbf{a}) \equiv 0$ in $\Sigma^{\varepsilon}$ since $\mathbf{w}_{k} \in \mathcal{R}\left(Q_{k}\right)$, where $\mathcal{E}_{\mathbf{x}}$ denotes the $2 \times 2$ absolutely continuous symmetric gradient with respect to the $n-1$ variables $\mathbf{x}$ only. In addition $S_{\mathbf{a}}, S_{\mathbf{b}}$ and $S_{\mathbf{m}}$ are subsets of $S=\partial_{l} Q_{k} \cap \Sigma^{\varepsilon} \cap\{z=0\}$ and $S_{\mathbf{w}} \subset S \otimes(-\varepsilon, \varepsilon) \stackrel{\text { def }}{=} S^{\varepsilon}$.

We may apply property BD6 in the $(n-1)$-dimensional cube $\Sigma$ taking into account Lemma 2.3 and the relationship between Sobolev exponents $1^{*}(n)<1^{*}(n-1)$ : since $\mathcal{E}_{\mathbf{x}}(\mathbf{a}) \equiv 0$ we obtain

$$
\begin{aligned}
\|\mathbf{a}\|_{L^{n / n-1}(\Sigma)} & \leqslant c \int_{S}|[\mathbf{a}]| \mathrm{d} \mathcal{H}^{n-2}(\mathbf{x}) \\
\|\mathbf{b}\|_{L^{n / n-1}(\Sigma)} & \leqslant c \int_{S}|[\mathbf{b}]| \mathrm{d} \mathcal{H}^{n-2}(\mathbf{x}) \\
\|\mathbf{m}\|_{L^{n / n-1}(\Sigma)} & \leqslant c \int_{S}|[\mathbf{m}]| \mathrm{d} \mathcal{H}^{n-2}(\mathbf{x}) .
\end{aligned}
$$

By (2.12)-(2.14) and Minkowski inequality we get

$$
\begin{aligned}
\|\mathbf{w}\|_{L^{\frac{n}{n-1}\left(\Sigma^{\varepsilon}\right)}} & \leqslant c\left\{\int_{-\varepsilon}^{\varepsilon} \int_{\Sigma}\left(|\mathbf{a}|+|\varepsilon||\mathbf{b}|+\left|\mathbf{b} \cdot\left(\mathbf{x}-\mathbf{x}_{k}\right)\right|+|\mathbf{m}|\right)^{n /(n-1)} \mathrm{d} \mathbf{x} \mathrm{d} z\right\}^{\frac{n-1}{n}} \\
& \leqslant c \varepsilon^{(n-1) / n}\left\{\|\mathbf{a}\|_{L^{n /(n-1)}(\Sigma)}+\|\mathbf{b}\|_{L^{n /(n-1)}(\Sigma)}+\|\mathbf{m}\|_{L^{n /(n-1)}(\Sigma)}\right\} \\
& \leqslant c \varepsilon^{1-1 / n} \int_{S}(|[\mathbf{a}]|+|[\mathbf{b}]|+|[\mathbf{m}]|) \mathrm{d} \mathcal{H}^{n-2}(\mathbf{x}) .
\end{aligned}
$$

On the other hand, from (2.9) with $\tau=1 / 2$ Lemma 2.3 and (2.6) we get

$$
\begin{aligned}
\varepsilon^{2} \int_{S}(|[\mathbf{a}]|+|[\mathbf{b}]|+|[\mathbf{m}]|) \mathrm{d} \mathcal{H}^{n-2}(\mathbf{x}) \\
\quad \leqslant c \int_{S}\left(\int_{\varepsilon}^{\varepsilon}(|[\mathbf{B}(\mathbf{x})]|+|[\mathbf{a}+z \mathbf{b}]|+|[\mathbf{m}]|) \mathrm{d} z\right) \mathrm{d} \mathcal{H}^{n-2}(\mathbf{x}) \\
\quad \leqslant c \int_{S^{\varepsilon}}(|[\mathbf{B}(\mathbf{x})]|+|[\mathbf{a}+z \mathbf{b}]|+|[\mathbf{m}]|) \mathrm{d} \mathcal{H}^{n-1}(\mathbf{X}) \leqslant c|\mathrm{e}(\mathbf{w})|\left(\Sigma^{\varepsilon}\right)
\end{aligned}
$$

and inequality (2.11) follows now by recalling Lemma 2.3 and joining (2.15) with (2.16) times $\varepsilon^{-1-1 / n}$.

Proof of Theorem 2.1. Let now $\mathbf{v} \in B D\left(\Sigma^{\varepsilon}\right)$ with spt $\mathbf{v} \subset \overline{\Omega^{\varepsilon}}$. We choose rigid displacements $\mathbf{w}_{k}$ as in Lemma 2.4 such that (2.8) holds in each $Q_{k}$, with the choice $s=n /(n-1)$. In particular, we 
choose $\mathbf{w}_{k} \equiv 0$ in each $\mathbf{Q}_{k}$ s.t. $\mathbf{Q}_{k} \cap \Omega^{\varepsilon}=\emptyset$. By Minkowski inequality and (2.6), (2.7) we get

$$
\begin{aligned}
\|\mathbf{v}\|_{L^{n /(n-1)}\left(\Omega^{\varepsilon}\right)} & \leqslant\left(\sum_{k}\|\mathbf{v}-\mathbf{w}\|_{L^{n /(n-1)}\left(Q_{k}\right)}\right)+\|\mathbf{w}\|_{L^{n /(n-1)}\left(\Omega^{\varepsilon}\right)} \\
& \leqslant c \sum_{k}|\mathrm{e}(\mathbf{v})|\left(Q_{k}\right)+\frac{c}{\varepsilon^{(n+1) / n}} \int_{S^{\varepsilon}}|[\mathbf{w}]| \\
& \leqslant c|\mathrm{e}(\mathbf{v})|\left(S^{\varepsilon}\right)+\frac{c}{\varepsilon^{(n+1) / n}}\left(\int_{S^{\varepsilon}}\left|\mathbf{w}^{+}-\mathbf{v}^{+}\right|+\left|\mathbf{w}^{-}-\mathbf{v}^{-}\right|+|[\mathbf{v}]|\right) .
\end{aligned}
$$

By (2.6), (2.8), taking into account that $\int_{S^{\varepsilon}}|[\mathbf{v}]| \leqslant|\mathrm{e}(\mathbf{v})|\left(\Sigma^{\varepsilon}\right)$, we get

$$
\begin{aligned}
& |\mathrm{e}(\mathbf{v})|\left(\Sigma^{\varepsilon}\right)+c \varepsilon^{-1-\frac{1}{n}}\left(\int_{S^{\varepsilon}}\left|\mathbf{w}^{+}-\mathbf{v}^{+}\right|+\left|\mathbf{w}^{-}-\mathbf{v}^{-}\right|+|[\mathbf{v}]|\right) \\
& \leqslant c \varepsilon^{-2-\frac{1}{n}} \sum_{k} \int_{Q_{k}}|\mathbf{w}-\mathbf{v}|+c \varepsilon^{-1-\frac{1}{n}}|\mathrm{e}(\mathbf{v})|\left(\Sigma^{\varepsilon}\right) \\
& \leqslant c \varepsilon^{-1-\frac{1}{n}}|\mathrm{e}(\mathbf{v})|\left(\Sigma^{\varepsilon}\right) .
\end{aligned}
$$

We obtain (2.4) by gathering the previous inequalities together. Inequality (2.5) follows from Hölder inequality.

EXAMPLE 2.5 The rates $\varepsilon^{-1-1 / n}$ and $\varepsilon^{\frac{1}{s}-2}$ in (2.5) are optimal since, if $n \geqslant 2$, and for $\mathbf{x} \in \Sigma$, $z \in(-\varepsilon, \varepsilon)$, we can choose $\varphi \in C^{2}(\Sigma)$ with $\varphi \not \equiv 0$, spt $\varphi \subset \Omega$ and set

$$
\mathbf{v}(\mathbf{x}, z)=(-z D \varphi(\mathbf{x}), \varphi(\mathbf{x}))^{T} .
$$

Clearly, spt $\mathbf{v} \subset \Omega^{\varepsilon}, \mathbf{v} \in S B D\left(\Sigma^{\varepsilon}\right), J_{\mathbf{v}^{\varepsilon}}=\emptyset$ and $\mathcal{E}(\mathbf{v})=\left(\begin{array}{cc}-z D^{2} \varphi & \mathbf{0} \\ \mathbf{0}^{T} & 0\end{array}\right)$. Therefore,

$$
\int_{\Sigma^{\varepsilon}}|\mathrm{e}(\mathbf{v})|=\int_{\Sigma^{\varepsilon}}|\mathcal{E}(\mathbf{v})| \mathrm{d} \mathbf{x}=\varepsilon^{2}\left\|D^{2} \varphi\right\|_{L^{1}(\Sigma)}
$$

while, as $\varepsilon \rightarrow 0_{+}$,

$$
\left(\int_{\Sigma^{\varepsilon}}|\mathbf{v}|^{s}\right)^{\frac{1}{s}} \sim \varepsilon^{\frac{1}{s}} 2^{\frac{1}{s}}\|\varphi\|_{L^{s}(\Sigma)}
$$

which shows that the estimates (2.4), (2.5) are optimal.

\section{Approximation of the linear elastic plastic plate (LP)}

From now on we deal with the physical situation, say $n=3, \mathbf{x}=(x, y)$, and we assume

$$
\begin{gathered}
\Omega \subset \mathbb{R}^{2} \text { is a bounded connected Lipschitz open set } \\
\bar{\Omega} \subset \Sigma=(-L, L)_{x} \times(-L, L)_{y}
\end{gathered}
$$

and we set, $\forall \varepsilon \in(0,1]$,

$$
\Omega^{\varepsilon}=\Omega \times(-\varepsilon, \varepsilon), \quad \Sigma^{\varepsilon}=\Sigma \times(-\varepsilon, \varepsilon), \quad \Gamma=\partial \Omega, \quad \Gamma^{\varepsilon}=\Gamma \times(-\varepsilon, \varepsilon) .
$$


We denote with $\mathbf{x}=(x, y)$ the variables on $\Omega$, with $z$ the variable on $(-\varepsilon, \varepsilon)$ and with $t$ the re-scaled vertical variable variable on $(-1,1)$. For every vector field $\mathbf{W}: \Omega^{1}\left(\right.$ respectively $\left.\Omega^{\varepsilon}\right) \rightarrow \mathbf{R}^{3}$ we denote with $\mathbf{w}=\left(w_{1}, w_{2}\right)$ its two horizontal components, with $w_{3}$ its vertical one and for every $\mathbf{X} \in \Omega^{1}\left(\right.$ resp. $\left.\Omega^{\varepsilon}\right)$ we set $\mathbf{X}=(\mathbf{x}, t)$ and $\mathbf{X}=(\mathbf{x}, z)$ with $\mathbf{x}=(x, y) \in \Sigma, t \in(-1,1)$ and $z \in(-\varepsilon, \varepsilon)$ respectively.

$\Omega^{\varepsilon}$ will be the reference configuration of a thick elastic plastic body which we will assume loaded by a dead force field $\mathbf{F}^{\varepsilon}$, not necessarily perpendicular to the middle surface $\Omega$, such that

$$
\mathbf{F}^{\varepsilon}(x, y)=\frac{\varepsilon^{2}}{2} \mathbf{G}(x, y) \quad \mathbf{G}=\left(g_{1}, g_{2}, g\right) \in L^{p}\left(\Omega, \mathbf{R}^{3}\right), p \in[3,+\infty] .
$$

Without relabelling we denote by $\mathbf{F}^{\varepsilon}$ and $\mathbf{G}$ also their trivial extensions on $\Sigma^{\varepsilon}$ (say spt $F^{\varepsilon}$, spt $G \subset$ $\left.\overline{\Omega^{\varepsilon}}\right)$.

We assume that the stored energy due to a displacement $\mathbf{V} \in \operatorname{SBD}\left(\Sigma^{\varepsilon}\right)$ is given by

$$
\mathcal{G}^{\varepsilon}(\mathbf{V})=\int_{\Sigma^{\varepsilon}}\left(\mu|\mathcal{E}(\mathbf{V})|^{2}+\frac{\lambda}{2}|\operatorname{Tr} \mathcal{E}(\mathbf{V})|^{2}\right) \mathrm{d} \mathbf{X}+\int_{J_{\mathbf{V}}} \theta^{\varepsilon}\left([\mathbf{V}], \nu_{\mathbf{V}}\right) \mathrm{d} \mathcal{H}^{2}(\mathbf{X})
$$

where $\lambda, \mu$ are the Lamé constants, with

$$
\begin{gathered}
\mu>0, \quad 2 \mu+3 \lambda>0, \quad \delta>0, \quad \gamma>0 \\
\theta^{\varepsilon}(\eta, \xi)=\varepsilon^{2} \delta|\xi|+\varepsilon \gamma|\eta \odot \xi| .
\end{gathered}
$$

More explicitly $\int_{J_{\mathbf{V}}} \theta^{\varepsilon}\left([\mathbf{V}], \nu_{\mathbf{V}}\right) \mathrm{d} \mathcal{H}^{2}(\mathbf{X})=\varepsilon^{2} \delta \mathcal{H}^{2}\left(J_{\mathbf{V}}\right)+\varepsilon \gamma \int_{J_{\mathbf{V}}}\left|[\mathbf{V}] \odot \nu_{\mathbf{V}}\right| \mathrm{d} \mathcal{H}^{2}(\mathbf{X})$.

Now we introduce the load energy and the total energy associated to the displacement field $\mathbf{V}$ :

$$
\begin{gathered}
\mathcal{L}^{\varepsilon}(\mathbf{V})=\int_{\Sigma^{\varepsilon}} \mathbf{F}^{\varepsilon} \cdot \mathbf{V} \mathrm{d} \mathbf{X}=\frac{\varepsilon^{2}}{2} \int_{\Sigma^{\varepsilon}} \mathbf{G} \cdot \mathbf{V} \mathrm{d} \mathbf{X} \\
\mathcal{F}^{\varepsilon}(\mathbf{V})=\mathcal{G}^{\varepsilon}(\mathbf{V})-\mathcal{L}^{\varepsilon}(\mathbf{V})
\end{gathered}
$$

and we state the weak formulation for the Dirichlet problem (see also [9])

$$
\min \left\{\mathcal{F}^{\varepsilon}(\mathbf{V}): \mathbf{V} \in \operatorname{SBD}\left(\Sigma^{\varepsilon}\right), \operatorname{spt} \mathbf{V} \subset \overline{\Omega^{\varepsilon}}\right\} .
$$

THEOREM 3.1 Assume (3.1)-(3.9) and

$$
\|\mathbf{G}\|_{L^{p}(\Omega)}<\frac{\sqrt[3]{4} \gamma|\Sigma|^{\frac{3-p}{3 p}}}{C_{\Omega}}
$$

where $C_{\Omega}$ is the constant of Korn-Poincaré inequality (2.4) of Lemma 2.1.

Then the problem $\mathbf{L} \mathbf{P}^{\varepsilon}$ achieves a finite minimum.

We notice that a smallness condition on loads, like the safe load condition (3.10), is not only sufficient but also necessary in order to find solutions of problem $\mathbf{L} \mathbf{P}^{\varepsilon}$, as usual in variational problems with linear growth (see [6]). 
Proof of Theorem 3.1. $\mathcal{F}^{\varepsilon}$ is seq. $w^{*} B D$ 1.s.c. (see [9]). We apply the same method of Theorem 3.3 in [37] with $n=3$ (see [6]) in the set $\Sigma^{\varepsilon}, a=\varepsilon^{2} \delta, b=\varepsilon \gamma$. By Theorem 2.1, we get $K_{\Sigma^{\varepsilon}}=$ $C_{\Omega} \varepsilon^{-4 / 3}$, say

$$
\|\mathbf{v}\|_{L^{n^{\prime}}\left(\Omega^{\varepsilon}\right)} \leqslant K_{\Sigma^{\varepsilon}}|\mathrm{e}(\mathbf{v})|\left(\Sigma^{\varepsilon}\right) \quad \forall \varepsilon \in(0,1), \forall \mathbf{v} \in S B D\left(\Sigma^{\varepsilon}\right): \operatorname{spt} \mathbf{v} \subset \overline{\Sigma^{\varepsilon}} .
$$

Hence

$$
\begin{aligned}
\left\|\mathbf{F}^{\varepsilon}\right\|_{L^{p}\left(\Omega^{\varepsilon}\right)} & =\left\|\frac{\varepsilon^{2}}{2} \mathbf{G}\right\|_{L^{p}\left(\Omega^{\varepsilon}\right)}=2^{\frac{1}{p}-1} \varepsilon^{2+\frac{1}{p}}\|\mathbf{G}\|_{L^{p}(\Omega)} \\
& \stackrel{(3.10)}{<} \frac{\gamma \varepsilon}{\varepsilon^{-1-\frac{1}{p}} C_{\Omega}(2|\Sigma|)^{\frac{1}{3}-\frac{1}{p}}}=\frac{b}{\varepsilon^{-4 / 3} C_{\Omega}(2 \varepsilon|\Sigma|)^{\frac{1}{3}-\frac{1}{p}}}=\frac{b}{K_{\Sigma^{\varepsilon}\left|\Sigma^{\varepsilon}\right|^{\frac{p-3}{3 p}}}}
\end{aligned}
$$

that is (3.6') of [37] holds in this case.

From now on we will assume that Greek indices vary in the set $\{1,2\}$, and Roman indices in $\{1,2,3\}$, and we will denote with $\mathrm{e}_{\mathbf{x}}$ and $\mathcal{E}_{\mathbf{x}}$ the $2 \times 2$ tensor field whose components coincide with the horizontal components of e and $\mathcal{E}$ respectively.

For every $\mathbf{V} \in \operatorname{SBD}\left(\Sigma^{\varepsilon}\right)$ we define a vector field $\mathbf{U}$ in $\Sigma^{1}$ by a suitable re-scaling, and a family of functionals $E^{\varepsilon}$ to be evaluated on $\mathbf{U}$ (so that we can refer to a single fixed domain $\Sigma^{1}$ instead of varying domains $\Sigma^{\varepsilon}$ ):

$$
\begin{gathered}
\mathbf{U}(\mathbf{x}, t)=\left(\varepsilon^{-1} \mathbf{v}(\mathbf{x}, \varepsilon t), v_{3}(\mathbf{x}, \varepsilon t)\right), \quad \mathbf{x} \in \Sigma, z \in(-\varepsilon, \varepsilon), t \in(-1,1), \\
E^{\varepsilon}(\mathbf{U})=\varepsilon^{-3} \mathcal{F}^{\varepsilon}(\mathbf{V}) .
\end{gathered}
$$

We will see in Section 5 that actually such $\mathbf{U}$ belongs to $\operatorname{SBD}\left(\Sigma^{1}\right)$.

We fix $\mathbf{V}^{\varepsilon} \in \operatorname{argmin} \mathcal{F}^{\varepsilon}$ and $\mathbf{U}^{\varepsilon}(\mathbf{x}, t)=\left(\varepsilon^{-1} \mathbf{v}^{\varepsilon}(\mathbf{x}, \varepsilon t), v_{3}^{\varepsilon}(\mathbf{x}, \varepsilon t)\right)$. Obviously $\mathbf{U}^{\varepsilon} \in \operatorname{argmin} E^{\varepsilon}$ and $\operatorname{spt} \mathbf{U}^{\varepsilon} \subset \overline{\Omega^{\varepsilon}}$.

We study the asymptotic behaviour of the family $\mathbf{U}^{\varepsilon}$ as $\varepsilon \rightarrow 0_{+}$with respect to the strong convergence in $L^{1}\left(\Sigma^{1}\right)$ (denoted by $\sigma$ ). In order to describe the variational limit of functionals (3.11) it is useful to recall the notion of $\Gamma$ convergence (see $[21,22]$ ).

Definition 3.2 Let $\varepsilon>0,(S, \sigma)$ a complete metric space and $I, I^{\varepsilon}: S \rightarrow \mathbf{R} \cup\{+\infty\}$ a family of functionals. We say that

$$
\Gamma\left(\sigma^{-}\right) \lim _{\varepsilon \rightarrow 0^{+}} I^{\varepsilon}(s)=I(s)
$$

if and only if the two following conditions are satisfied:

$$
\begin{array}{llll}
\forall s \in S & \forall \varepsilon_{n} \rightarrow 0^{+} & \forall s_{n} \stackrel{\sigma}{\longrightarrow} s & \liminf _{n \rightarrow \infty} I^{\varepsilon_{n}}\left(s_{n}\right) \geqslant I(s), \\
\forall s \in S & \forall \varepsilon_{n} \rightarrow 0^{+} & \exists s_{n} \stackrel{\sigma}{\longrightarrow} s & \limsup _{n \rightarrow \infty} I^{\varepsilon_{n}}\left(s_{n}\right)=I(s) .
\end{array}
$$

The most important consequence of $\Gamma$-convergence is convergence of minimizers.

Theorem 3.3 ([22: Corollary 2.4 ]) Assume $I=\Gamma\left(\sigma^{-}\right) \lim _{\varepsilon \rightarrow 0^{+}} I^{\varepsilon}$. If $s^{\varepsilon} \in \operatorname{argmin} I^{\varepsilon}$ and $s_{\varepsilon} \stackrel{\sigma}{\longrightarrow} s$, then

$$
I^{\varepsilon}\left(s^{\varepsilon}\right) \rightarrow I(s)=\min \{I(s): s \in S\} .
$$


Now we can state the two main results of this section.

Theorem 3.4 Assume (3.1)-(3.9) and the safe load condition (3.10). Set

$\mathcal{A}=\left\{\mathbf{U} \in \operatorname{SBD}\left(\Sigma^{1}\right): \mathbf{U}=\left(\mathbf{u}, u_{3}\right), u_{3}=u_{3}(x, y), \operatorname{spt} u_{3} \subset \bar{\Omega}, \mathbf{u}(x, y, t)=\zeta(x, y)-t D u_{3}(x, y)\right\}$

$E^{0}(\mathbf{U})= \begin{cases}\int_{\Sigma}\left\{\frac{2}{3} \mu\left(\left|\nabla^{2} u_{3}\right|^{2}+\frac{\lambda}{\lambda+2 \mu}\left|\Delta^{a} u_{3}\right|^{2}\right)+2 \mu\left(\left|\mathcal{E}_{\mathbf{x}}(\zeta)\right|^{2}+\frac{\lambda}{\lambda+2 \mu}|\operatorname{div} \zeta|^{2}\right)\right\} \mathrm{d} x \mathrm{~d} y & \\ \quad+\int_{J_{\mathbf{U}}}\left(\delta+\gamma\left|[\mathbf{U}] \odot \nu_{\mathbf{U}}\right| \mathrm{d} \mathcal{H}^{2}(x, y, t)-\int_{\Sigma} g u_{3} \mathrm{~d} x \mathrm{~d} y\right. & \text { if } \mathbf{U} \in \mathcal{A} \\ +\infty & \text { otherwise. }\end{cases}$

Then

$$
\Gamma\left(L^{1}\left(\Sigma^{1}, \mathbb{R}^{3}\right)^{-}\right) \lim _{\varepsilon \rightarrow 0_{+}} E^{\varepsilon}(\mathbf{U})=E^{0}(\mathbf{U}) .
$$

REMARK 3.5 It is worth noticing that if $\mathbf{U}=\left(\mathbf{u}, u_{3}\right) \in \mathcal{A}$ then $\mathbf{u}(., t) \in \operatorname{SBD}(\Sigma)$ for a.e. $t \in$ $(-1,1)$ and hence $\zeta \in \operatorname{SBD}(\Sigma)$, spt $\zeta \subset \bar{\Omega}, \operatorname{spt} u_{3} \subset \bar{\Omega}, v_{\mathbf{U}}$ is a horizontal vector, say $\left(v_{\mathbf{U}}\right)_{3} \equiv 0 \mathcal{H}^{2}$ a.e. on $J_{\mathbf{U}}$, and $u_{3} \in \operatorname{SBH}(\Sigma)$, since

$$
\mathrm{e}(\mathbf{U})=\mathrm{e}(\mathbf{u})=\mathrm{e}(\zeta)-t \mathrm{e}\left(D u_{3}\right)=\mathrm{e}(\zeta)-t D^{2} u_{3} \in \mathcal{M}(\Sigma) \quad \text { a.e. } t
$$

whence $\mathrm{e}(\boldsymbol{\zeta})$ and $D^{2} u_{3}$ belong to $\mathcal{M}$ and, since $\mathrm{e}_{\mathbf{x}}(\mathbf{U})$ has no Cantor part, $u_{3} \in S B H, \zeta \in S B D$.

In particular, if $\mathbf{U}=\left(\mathbf{u}, u_{3}\right) \in \mathcal{A}$ and $E^{0}(\mathbf{U})<+\infty$, then $\nabla^{2} u_{3}=\nabla D u_{3} \in L^{2}$ and $\mathcal{E}(\zeta) \in L^{2}$.

In addition to Theorem 3.4 it is possible to prove the following result concerning convergence of minimizers of $\left(\mathbf{L} \mathbf{P}^{\varepsilon}\right)$.

Theorem 3.6 Assume (3.1)-(3.11). Then, $\forall \varepsilon \in(0,1]$, and for every $\mathbf{V}^{\varepsilon} \in \operatorname{argmin}\left(\mathbf{L} \mathbf{P}^{\varepsilon}\right)$, by setting $\mathbf{U}^{\varepsilon}(\mathbf{x}, t)=\left(\mathbf{u}^{\varepsilon}, u_{3}^{\varepsilon}\right)=\left(\varepsilon^{-1} \mathbf{v}^{\varepsilon}(\mathbf{x}, \varepsilon t), v_{3}^{\varepsilon}(\mathbf{x}, \varepsilon t)\right)=\left(\varepsilon^{-1} \mathbf{v}^{\varepsilon}(\mathbf{x}, z), v_{3}^{\varepsilon}(\mathbf{x}, z)\right)$, for $\mathbf{x} \in \Sigma, z \in$ $(-\varepsilon, \varepsilon), t \in(-1,1)$, we have, up to subsequences,

$$
\left(\mathbf{u}^{\varepsilon}, u_{3}^{\varepsilon}\right) \stackrel{w^{*}-S B D(\Sigma)}{\longrightarrow}\left(-t D u_{3}, u_{3}\right) \quad \text { and } \quad E^{\varepsilon}\left(\mathbf{U}^{\varepsilon}\right) \longrightarrow \mathcal{G}^{0}\left(u_{3}\right)
$$

for a suitable $u_{3}$ minimizer of

$$
\min \left\{\mathcal{G}^{0}(w): w \in \operatorname{SBH}(\Sigma), \operatorname{spt} w \subset \bar{\Omega}\right\}
$$

where

$$
\begin{aligned}
\mathcal{G}^{0}(w)= & \frac{2}{3} \mu \int_{\Sigma}\left(\left|\nabla^{2} w\right|^{2}+\frac{\lambda}{\lambda+2 \mu}\left|\Delta^{a} w\right|^{2}\right) \mathrm{d} \mathbf{x}+2 \delta \mathcal{H}^{1}\left(S_{D w}\right) \\
& +\gamma \int_{S_{D w}}|[D w]| \mathrm{d} \mathcal{H}^{1}(\mathbf{x})-\int_{\Sigma} g w \mathrm{~d} \mathbf{x} .
\end{aligned}
$$

We notice that (by denoting the Poisson ratio $v=\frac{\lambda}{2(\lambda+\mu)} \in(-1,1 / 2)$ and the stiffness coefficient $\left.T=\frac{4}{3} \mu \frac{\lambda+\mu}{\lambda+2 \mu}\right)$ the first integral in $\mathcal{G}^{0}$ recovers exactly the classic linear elastic plate energy in the undamaged region $\Sigma \backslash S_{D w}$ (see [34]):

$$
\begin{aligned}
\frac{2}{3} \mu \int_{\Sigma}\left(\left|\nabla^{2} w\right|^{2}+\frac{\lambda}{\lambda+2 \mu}\left|\Delta^{a} w\right|^{2}\right) \mathrm{d} \mathbf{x} & =\frac{1}{2} T \int_{\Sigma}\left((1-v)\left|\nabla^{2} w\right|^{2}+v\left|\Delta^{a} w\right|^{2}\right) \mathrm{d} \mathbf{x} \\
& =\frac{1}{2} T \int_{\Sigma}\left(\left|\Delta^{a} w\right|^{2}-2(1-v) \operatorname{det} \nabla^{2} w\right) \mathrm{d} \mathbf{x} .
\end{aligned}
$$


REMARK 3.7 The previous result makes precise in what sense the variational limit of the energies of $3 D$ thick bodies with damage at mesoscopic scale describes the energy of an elastic plastic plate: indeed, the limit functional takes into account other terms depending on $\zeta$ but, since $\zeta$ is un-coupled with $u_{3}$ in $E^{0}$, then the choice $\zeta \equiv 0$ is optimal. So that any minimizer $w$ of $\mathcal{G}^{0}$ leads to a minimizer $\mathbf{U}=(-t \nabla w, w)$ of $E^{0}$. The functional $E^{0}$ may have higher energy than $\mathcal{G}^{0}$ on competing vector fields, nevertheless $E^{0}$ has the same minimizers of $\mathcal{G}^{0}$ in (LP) (which describes the elastic plastic plate [14-16]), and the energy of minimizers are the same.

This fact is well known for a linearly elastic plate: here we show that, even in presence of free gradient discontinuities there is no shear in the limit problem as for the Kirchhoff-Love plate.

We conclude with a statement for equilibrium of an elastic plastic flat plate, clamped at the boundary and subject to transverse loads: actually a similar statement was already given ( [15: Theorem 4.1]); we observe that, with slightly stronger assumptions (e.g. $g \in L^{p}, p \geqslant 3$ ) in order to provide meaningful approximating problems in $B D$, the following Theorem 3.8 would be an easy consequence of Theorems 3.4, 3.6, since $E^{0}$ is the $\Gamma$ limit of equi-coercive functionals. Anyway, the direct proof is a straightforward application of the direct method in calculus of variation.

THEOREM 3.8 If $\mu>0,2 \mu+3 \lambda>0, \delta>0, \gamma>0$, and

$$
\|g\|_{\mathcal{M}(\Sigma)}<4 \gamma
$$

then $\mathbf{L P}$ achieves a finite minimum.

Proof. $\mathcal{G}^{0}$ is sequentially $w^{*}$ 1.s.c. in $S B H$. The quadratic growth with respect to the absolutely continuous part of $D^{2} w$ (due to $\mu>0,2 \mu+3 \lambda>0$ ) and the linear growth with coefficient $\gamma$ with respect to the singular part, together with inequalities (1.6) and (3.12), provide enough coercivity to compensate the load $g$. The support constraint is sequentially $w^{*}$ closed.

\section{Approximation of the rigid plastic slab (RS)}

Now we plug a divergent weight (which blows up as $\varepsilon \rightarrow 0^{+}$) in the first integral of (3.5): as a consequence we find a stiffer structure in the limit (see $[17,41])$.

Throughout this section we assume (3.1)-(3.4) and (3.6)-(3.8) and the safe load (3.10).

We assume that the stored energy due to a displacement $\mathbf{V}$ is given by

$$
\mathcal{T}^{\varepsilon}(\mathbf{V})=\varepsilon^{-1} \int_{\Sigma^{\varepsilon}}\left(\mu|\mathcal{E}(\mathbf{V})|^{2}+\frac{\lambda}{2}|\operatorname{Tr} \mathcal{E}(\mathbf{V})|^{2}\right) \mathrm{d} \mathbf{X}+\int_{J_{\mathbf{V}}} \theta^{\varepsilon}\left([\mathbf{V}], \nu_{\mathbf{V}}\right) \mathrm{d} \mathcal{H}^{2}(\mathbf{X}) .
$$

and, referring to (3.8), that the total mechanical energy is given by

$$
\Lambda^{\varepsilon}(\mathbf{V})=\mathcal{T}^{\varepsilon}(\mathbf{V})-\mathcal{L}^{\varepsilon}(\mathbf{V}) .
$$

Now we can state the weak formulation of the Dirichlet problem

$$
\min \left\{\Lambda^{\varepsilon}(\mathbf{V}): \mathbf{V} \in \operatorname{SBD}\left(\Sigma^{\varepsilon}\right), \operatorname{spt} \mathbf{V} \subset \overline{\Omega^{\varepsilon}}\right\} .
$$

THEOREM 4.1 Assume (3.1)-(3.4), (3.6), (3.8), (3.10), (4.1), (4.2) and $\varepsilon \in(0,1)$.

Then the problem $\mathbf{R S}^{\varepsilon}$ achieves a finite minimum. 
Proof. We argue as in the proof of Theorem 3.1.

For every $\mathbf{V} \in \operatorname{SBD}\left(\Sigma^{\varepsilon}\right)$ we define a vector field $\mathbf{U}$ in $\Sigma^{1}$ by a suitable re-scaling, and a family of functionals $\mathcal{W}^{\varepsilon}$ to be evaluated on $\mathbf{U}$ (so that we can refer to a single fixed domain $\Sigma^{1}$ ):

$$
\begin{gathered}
\mathbf{U}(\mathbf{x}, t)=\left(\varepsilon^{-1} \mathbf{v}(\mathbf{x}, \varepsilon t), v_{3}(\mathbf{x}, \varepsilon t)\right), \quad \mathbf{x} \in \Sigma, z \in(-\varepsilon, \varepsilon), t \in(-1,1), \\
\mathcal{W}^{\varepsilon}(\mathbf{U})=\varepsilon^{-3} \Lambda^{\varepsilon}(\mathbf{V}) .
\end{gathered}
$$

THEOREM 4.2 Assume (3.1)-(3.4), (3.6), (3.8), (3.10), (4.1)-(4.3). Set

$$
\begin{aligned}
& \mathcal{U}=\left\{\mathbf{U} \in \operatorname{SBD}\left(\Sigma^{1}\right):\right. \\
& \left.\mathbf{U}=\left(\mathbf{u}, u_{3}\right), u_{3}=u_{3}(x, y), \operatorname{spt} u_{3} \subset \bar{\Omega}, \mathbf{u}(x, y, t)=\zeta(x, y)-t D u_{3}(x, y), \mathcal{E}_{\mathbf{x}}(\zeta) \equiv \mathbf{0} \equiv \nabla D u_{3}\right\}, \\
& \mathcal{W}^{0}(\mathbf{U})= \begin{cases}\int_{J_{\mathbf{U}}}\left(\delta+\gamma\left|[\mathbf{U}] \odot v_{\mathbf{U}}\right| \mathrm{d} \mathcal{H}^{2}(x, y, t)-\int_{\Omega} g u_{3} \mathrm{~d} x \mathrm{~d} y\right. & \text { if } \mathbf{U} \in \mathcal{U}, \\
+\infty & \text { otherwise. }\end{cases}
\end{aligned}
$$

Then

$$
\Gamma\left(L^{1}\left(\Sigma^{1}, \mathbb{R}^{3}\right)^{-}\right) \lim _{\varepsilon \rightarrow 0_{+}} \mathcal{W}^{\varepsilon}=\mathcal{W}^{0} .
$$

Moreover, if $\mathbf{V}^{\varepsilon} \in \operatorname{argmin} \Lambda^{\varepsilon}$, then, up to subsequences,

$$
\mathbf{U}^{\varepsilon}=\left(\mathbf{u}^{\varepsilon}, u_{3}^{\varepsilon}\right) \stackrel{w^{*}-S B D(\Sigma)}{\longrightarrow}\left(-t D u_{3}, u_{3}\right) \quad \text { and } \quad \mathcal{W}^{\varepsilon}\left(\mathbf{U}^{\varepsilon}\right) \longrightarrow \mathcal{T}^{0}\left(u_{3}\right)
$$

for a suitable minimizer $u_{3}$ of

$$
\min \left\{\mathcal{T}^{0}(w): w \in \operatorname{SBH}(\Sigma), \operatorname{spt} w \subset \bar{\Omega}, \nabla D w \equiv 0\right\}
$$

where

$$
\mathcal{T}^{0}(w)=2 \delta \mathcal{H}^{1}\left(S_{D w}\right)+\gamma \int_{S_{D w}}|[D w]| \mathrm{d} \mathcal{H}^{1}(\mathbf{x})-\int_{\Sigma} g w \mathrm{~d} \mathbf{x} .
$$

By arguing as in Theorem 3.8 one can prove the following statement.

THEOREM 4.3 If $\delta>0, \gamma>0$, and (3.12) holds true, then RS achieves a finite minimum.

\section{Proof of convergence results}

In this section we prove Theorems 3.4, 3.6 and 4.2.

The proof of Theorem 3.5 (approximation of the elastic plastic plate) will proceed in two steps: in the first one we give a lower bound for the $\Gamma$ liminf and in the second one we obtain an upper bound for the $\Gamma$ lim sup. We remark explicitly that both estimates are obtained without using any abstract result of representation for the $\Gamma$ limit. Then we deal with convergence of minimizers by proving Theorem 3.6, and eventually the approximation of the rigid plastic plate (Theorem 4.2) is discussed.

Throughout this section $\Gamma$ denotes $\Gamma\left(\sigma^{-}\right)$where $\sigma$ is the strong topology of $L^{1}\left(\Sigma^{1}, \mathbb{R}^{3}\right)$. 
Proof of Theorem 3.4 Step 1: lower bound for the $\Gamma$ limit.

For every $\mathbf{V}=\left(\mathbf{v}, v_{3}\right) \in S B D\left(\Sigma^{\varepsilon}\right)$, we define a vector field in $\Sigma^{1}$ by $\mathbf{U}(\mathbf{x}, t)=$ $\left(\varepsilon^{-1} \mathbf{v}(\mathbf{x}, \varepsilon t), v_{3}(\mathbf{x}, \varepsilon t)\right)$ and we set

$$
\begin{cases}\widetilde{\mathrm{e}}_{\alpha \beta}(\mathbf{U}) \stackrel{\text { def }}{=} \mathrm{e}_{\alpha \beta}(\mathbf{V})=\frac{\varepsilon}{2}\left(\frac{\partial u_{\alpha}}{\partial x_{\beta}}+\frac{\partial u_{\beta}}{\partial x_{\alpha}}\right) & x_{\alpha}, x_{\beta}=x, y \\ \widetilde{\mathrm{e}}_{\alpha t}(\mathbf{U}) \stackrel{\text { def }}{=} \mathrm{e}_{\alpha z}(\mathbf{V})=\frac{1}{2}\left(\frac{\partial u_{\alpha}}{\partial t}+\frac{\partial u_{3}}{\partial x_{\alpha}}\right) & x_{\alpha}=x, y \\ \widetilde{\mathrm{e}}_{t t}(\mathbf{U}) \stackrel{\text { def }}{=} \mathrm{e}_{z z}(\mathbf{V})=\varepsilon^{-1 \frac{\partial u_{3}}{\partial t} .} & \end{cases}
$$

Since $\mathbf{V} \in \operatorname{SBD}\left(\Sigma^{\varepsilon}\right)$ we deduce that $\widetilde{\mathrm{e}}(\mathbf{U})$ is a measure without Cantor part and therefore $\mathrm{e}(\mathbf{U})$ is a measure without Cantor part too. Then $\mathbf{U} \in \operatorname{SBD}\left(\Sigma^{1}\right)$ but $\mathbf{U}$ does not necessarily belong to $\mathcal{A}$. So we can define

$$
\begin{cases}\widetilde{\mathcal{E}}_{\alpha \beta}(\mathbf{U}) \stackrel{\text { def }}{=} \mathcal{E}_{\alpha \beta}(\mathbf{V})=\frac{\varepsilon}{2}\left(\nabla_{\beta} u_{\alpha}+\nabla_{\alpha} u_{\beta}\right) & x_{\alpha}, x_{\beta}=x, y \\ \widetilde{\mathcal{E}}_{\alpha t}(\mathbf{U}) \stackrel{\text { def }}{=} \mathcal{E}_{\alpha z}(\mathbf{V})=\frac{1}{2}\left(\nabla_{t} u_{\alpha}+\nabla_{\alpha} u_{3}\right) & x_{\alpha},=x, y \\ \widetilde{\mathcal{E}}_{t t}(\mathbf{U}) \stackrel{\text { def }}{=} \mathcal{E}_{z z}(\mathbf{V})=\varepsilon^{-1} \nabla_{t} u_{3} . & \end{cases}
$$

Moreover, the change of variables

$$
(x, y, z) \rightarrow(x, y, t)=(x, y, z / \varepsilon)
$$

carries $J_{\mathbf{V}}$ onto $J_{\mathbf{U}}$, hence, by setting here and in the following $\overline{\nu_{\mathbf{U}}}=\left(\left(v_{\mathbf{U}}\right)_{1},\left(\nu_{\mathbf{U}}\right)_{2}\right)$, we get

$$
\nu_{\mathbf{U}}=\mathcal{S}^{-1}\left(\varepsilon^{-1 \overline{\nu_{\mathbf{V}}}},\left(\nu_{\mathbf{V}}\right)_{3}\right) \quad \text { where } \quad \mathcal{S}=\sqrt{\varepsilon^{-2}\left|\overline{\nu_{\mathbf{V}}}\right|^{2}+\left|\left(\nu_{\mathbf{V}}\right)_{3}\right|^{2}}
$$

e.g. $\left(v_{\mathbf{V}}\right)_{3}=0$ entails $\nu_{\mathbf{U}}=v_{\mathbf{V}}$, while if $\left(\nu_{\mathbf{V}}\right)_{3} \neq 0$ then $\nu_{\mathbf{U}}$ is squeezed on the $x, y$ plane as $\varepsilon \rightarrow 0$.

By the area formula (see [30: Theorem 3.2.22(3)]) we get

$$
\mathrm{d} \mathcal{H}^{2}(x, y, z)\left\llcorner J_{\mathbf{V}}=\mathcal{S}^{-1} \mathrm{~d} \mathcal{H}^{2}(x, y, t)\left\llcorner J_{\mathbf{U}}\right.\right.
$$

and since $\theta^{\varepsilon}(\eta,$.$) is 1-homogeneous we get$

$$
\begin{aligned}
\int_{J_{\mathbf{V}}} & \theta^{\varepsilon}\left([\mathbf{V}], \nu_{\mathbf{V}}\right) \mathrm{d} \mathcal{H}^{2}(x, y, z) \\
& =\int_{J_{\mathbf{U}}} \theta^{\varepsilon}\left(\varepsilon\left[u_{1}\right], \varepsilon\left[u_{2}\right],\left[u_{3}\right], \varepsilon \mathcal{S}\left(\overline{\nu_{\mathbf{U}}}\right)_{1}, \varepsilon \mathcal{S}\left(\overline{\nu_{\mathbf{U}}}\right)_{2}, \mathcal{S}\left(\nu_{\mathbf{U}}\right)_{3}\right) \mathcal{S}^{-1} \mathrm{~d} \mathcal{H}^{2}(x, y, t) \\
& =\varepsilon \int_{J_{\mathbf{U}}} \theta^{\varepsilon}\left(\varepsilon\left[u_{1}\right], \varepsilon\left[u_{2}\right],\left[u_{3}\right],\left(\overline{\nu_{\mathbf{U}}}\right)_{1},\left(\overline{\nu_{\mathbf{U}}}\right)_{2}, \varepsilon^{-1}\left(\nu_{\mathbf{U}}\right)_{3}\right) \mathrm{d} \mathcal{H}^{2}(x, y, t) .
\end{aligned}
$$

By formulae (5.1)-(5.3), (3.4), (3.8) the functional $\mathcal{F}^{\varepsilon}(\mathbf{V})$ may be rewritten in terms of $\mathbf{U}$ as follows:

$$
\begin{aligned}
\mathcal{F}^{\varepsilon}(\mathbf{V})= & \varepsilon \int_{\Sigma^{1}}\left(\mu|\widetilde{\mathcal{E}}(\mathbf{U})|^{2}+\frac{\lambda}{2}|\operatorname{Tr} \widetilde{\mathcal{E}}(\mathbf{U})|^{2}\right) \mathrm{d} \mathbf{x} \mathrm{d} t \\
& +\varepsilon \int_{J_{\mathbf{U}}} \theta^{\varepsilon}\left(\varepsilon[\mathbf{u}],\left[u_{3}\right], \overline{\nu_{\mathbf{U}}}, \varepsilon^{-1}\left(\nu_{\mathbf{U}}\right)_{3}\right) \mathrm{d} \mathcal{H}^{2}(\mathbf{x}, t) \\
& -\frac{\varepsilon^{3}}{2} \int_{\Sigma^{1}} g \cdot u_{3} \mathrm{~d} \mathbf{x} \mathrm{d} t-\frac{\varepsilon^{4}}{2} \int_{\Sigma^{1}} \mathbf{g} \cdot \mathbf{u} \mathrm{d} \mathbf{x} \mathrm{d} t
\end{aligned}
$$


From now on we will use the notation $v(\mathbf{W})$ instead of $v_{\mathbf{W}}$ whenever the label $\mathbf{W}$ cannot be read easily when written as an index.

We have to prove that given $\mathbf{U}, \mathbf{U}^{\varepsilon} \in S B D\left(\Sigma^{\varepsilon}\right)$, with $\varepsilon \in(0,1)$, and $\mathbf{U}^{\varepsilon} \rightarrow \mathbf{U}$ in $L^{1}\left(\Sigma^{1}\right)$, then $\liminf _{\varepsilon \rightarrow 0^{+}} E^{\varepsilon}\left(\mathbf{U}^{\varepsilon}\right) \geqslant E^{0}(\mathbf{U})$.

We may assume that $E^{\varepsilon}\left(\mathbf{U}^{\varepsilon}\right) \leqslant c<+\infty$ otherwise the inequality is trivial. So we fix, $\forall \varepsilon \in$ $(0,1), \mathbf{V}^{\varepsilon}=\left(\mathbf{v}^{\varepsilon}, v_{3}^{\varepsilon}\right) \in S B D\left(\Sigma^{\varepsilon}\right)$, with $\mathbf{U}^{\varepsilon}=\left(\mathbf{u}^{\varepsilon}, u_{3}^{\varepsilon}\right)=\left(\varepsilon^{-1} \mathbf{v}^{\varepsilon}(\mathbf{x}, \varepsilon t), v_{3}^{\varepsilon}(\mathbf{x}, \varepsilon t)\right.$ such that

$$
\mathbf{U}^{\varepsilon} \rightarrow \mathbf{U} \quad \text { in } L^{1}\left(\Sigma^{1}\right), \quad E^{\varepsilon}\left(\mathbf{U}^{\varepsilon}\right) \leqslant c .
$$

By $E^{\varepsilon}\left(\mathbf{U}^{\varepsilon}\right)=\varepsilon^{-3} \mathcal{F}^{\varepsilon}\left(\mathbf{V}^{\varepsilon}\right)$ we get $\mathcal{F}^{\varepsilon}\left(\mathbf{V}^{\varepsilon}\right) \leqslant c \varepsilon^{3}$. Then by identity $\mathbf{U}(\mathbf{x}, t)=$ $\left(\varepsilon^{-1} \mathbf{v}^{\varepsilon}(\mathbf{x}, \varepsilon t), v_{3}^{\varepsilon}(\mathbf{x}, \varepsilon t)\right),(2.5)$, BD5-BD7, Lemma 2.3, Hölder and Young inequalities, we get, for small $\varepsilon$,

$$
\begin{aligned}
& \mathcal{G}^{\varepsilon}\left(\mathbf{V}^{\varepsilon}\right) \leqslant c \varepsilon^{3}+\frac{\varepsilon^{2}}{2} \int_{\Sigma^{\varepsilon}} \mathbf{G} \cdot \mathbf{V}^{\varepsilon} \mathrm{d} x \mathrm{~d} y \leqslant c \varepsilon^{3}+2^{(1-p) / p} \varepsilon^{2+1 / p}\|\mathbf{G}\|_{L^{p}(\Omega)}\left\|\mathbf{V}^{\varepsilon}\right\|_{L^{p^{\prime}}\left(\Sigma^{\varepsilon}\right)} \\
& \leqslant c \varepsilon^{3}+C_{\Omega} 2^{-2 / 3} \varepsilon|\Omega|^{\frac{p-3}{3 p}}\|\mathbf{G}\|_{L^{p}(\Omega)}\left\{\int_{\Sigma^{\varepsilon}}\left|\mathcal{E}\left(\mathbf{V}^{\varepsilon}\right)\right| \mathrm{d} \mathbf{X}+\int_{J_{\mathbf{V}^{\varepsilon}}} \mid\left[\mathbf{V}^{\varepsilon} \odot v\left(\mathbf{V}^{\varepsilon}\right] \mid \mathrm{d} \mathcal{H}^{2}(\mathbf{X})\right\}\right. \\
& \leqslant c \varepsilon^{3}+C_{\Omega} 2^{-1 / 6}|\Sigma|^{\frac{5 p-6}{6 p}} \varepsilon^{\frac{3}{2}}\|\mathbf{G}\|_{L^{p}(\Omega)}\left(\int_{\Sigma^{\varepsilon}}\left|\mathcal{E}\left(\mathbf{V}^{\varepsilon}\right)\right|^{2} \mathrm{~d} \mathbf{X}\right)^{1 / 2} \\
& \quad+C_{\Omega} 2^{-2 / 3} \varepsilon|\Sigma|^{\frac{p-3}{3 p}}\|\mathbf{G}\|_{L^{p}(\Omega)} \int_{J_{\mathbf{V}^{\varepsilon}}}\left|\left[\mathbf{V}^{\varepsilon} \odot v\left(\mathbf{V}^{\varepsilon}\right)\right]\right| \mathrm{d} \mathcal{H}^{2}(\mathbf{X}) \\
& \leqslant c \varepsilon^{3}+\frac{\mu}{6} \int_{\Sigma^{\varepsilon}}\left|\mathcal{E}\left(\mathbf{V}^{\varepsilon}\right)\right|^{2} \mathrm{~d} \mathbf{X}+\varepsilon\left(C_{\Omega} 2^{-2 / 3}|\Sigma|^{\frac{p-3}{3 p}}\|\mathbf{G}\|_{L^{p}(\Omega)}\right) \int_{J_{\mathbf{V}^{\varepsilon}}}\left|\left[\mathbf{V}^{\varepsilon} \odot v\left(\mathbf{V}^{\varepsilon}\right)\right]\right| \mathrm{d} \mathcal{H}^{2}(\mathbf{X})
\end{aligned}
$$

Taking into account that, by $|\operatorname{Tr} A|^{2} \leqslant 3|A|^{2}$ and $\lambda^{-}<\frac{2}{3} \mu$,

$$
\mathcal{G}^{\varepsilon}\left(\mathbf{V}^{\varepsilon}\right) \geqslant \frac{\mu}{3} \int_{\Sigma^{\varepsilon}}\left|\mathcal{E}\left(\mathbf{V}^{\varepsilon}\right)\right|^{2} \mathrm{~d} \mathbf{X}+\varepsilon \gamma \int_{J_{\mathbf{V}^{\varepsilon}}}\left|\left[\mathbf{V}^{\varepsilon} \odot v\left(\mathbf{V}^{\varepsilon}\right)\right]\right| \mathrm{d} \mathcal{H}^{2}(\mathbf{X})+\varepsilon^{2} \delta \mathcal{H}^{2}\left(J_{\mathbf{V}^{\varepsilon}}\right)
$$

the safe load condition (3.10) and (3.7), (5.3), (5.6) yield

$$
\int_{\Sigma^{\varepsilon}}\left|\mathcal{E}\left(\mathbf{V}^{\varepsilon}\right)\right|^{2} \mathrm{~d} \mathbf{x} \mathrm{d} z+\varepsilon \int_{J_{\mathbf{U}^{\varepsilon}}} \theta^{\varepsilon}\left(\varepsilon\left[u_{1}^{\varepsilon}\right], \varepsilon\left[u_{2}^{\varepsilon}\right],\left[u_{3}^{\varepsilon}\right],\left(v_{\mathbf{U}^{\varepsilon}}\right)_{1},\left(v_{\mathbf{U}^{\varepsilon}}\right)_{2}, \varepsilon^{-1}\left(v_{\mathbf{U}^{\varepsilon}}\right)_{3}\right) \mathrm{d} \mathcal{H}^{2}(x, y, t) \leqslant C \varepsilon^{3}
$$

and, by (5.4),

$$
\int_{\Sigma^{1}}\left|\widetilde{\mathcal{E}}\left(\mathbf{U}^{\varepsilon}\right)\right|^{2} \mathrm{~d} \mathbf{x} \mathrm{d} t+\int_{J_{\mathbf{U}^{\varepsilon}}} \theta^{\varepsilon}\left(\varepsilon\left[u_{1}^{\varepsilon}\right], \varepsilon\left[u_{2}^{\varepsilon}\right],\left[u_{3}^{\varepsilon}\right],\left(v_{\mathbf{U}^{\varepsilon}}\right)_{1},\left(v_{\mathbf{U}^{\varepsilon}}\right)_{2}, \varepsilon^{-1}\left(v_{\mathbf{U}^{\varepsilon}}\right)_{3}\right) \mathrm{d} \mathcal{H}^{2}(x, y, t) \leqslant C \varepsilon^{2} .
$$


Then, by using Lemma 2.3 we obtain now

$$
\begin{gathered}
\int_{J_{\mathbf{U}^{\varepsilon}}}\left|\left[u_{3}^{\varepsilon}\right]\left(\nu_{\mathbf{U}^{\varepsilon}}\right)_{3}\right| \mathrm{d} \mathcal{H}^{2} \leqslant c \varepsilon^{2} ; \\
\int_{\Sigma^{1}}\left|\widetilde{\mathcal{E}}\left(\mathbf{U}^{\varepsilon}\right)\right|^{2} \mathrm{~d} \mathbf{x} \mathrm{d} t \leqslant c \varepsilon^{2} \\
\int_{\Sigma^{1}}\left|\nabla_{t} u_{3}^{\varepsilon}\right|^{2} \mathrm{~d} \mathbf{x} \mathrm{d} t \leqslant c \varepsilon^{4} \\
\int_{J_{\mathbf{U}^{\varepsilon}}}\left|\left[\mathbf{U}_{\alpha}^{\varepsilon}\right]\left(\nu_{\mathbf{U}^{\varepsilon}}\right)_{3}+\left[u_{3}^{\varepsilon}\right]\left(\nu_{\mathbf{U}^{\varepsilon}}\right)_{\alpha}\right| \mathrm{d} \mathcal{H}^{2} \leqslant c \varepsilon ; \quad(\alpha=1,2) \\
\int_{J_{\mathbf{U}^{\varepsilon}}}\left(1+\left|\left[\mathbf{U}^{\varepsilon}\right]\right|\right) \leqslant C .
\end{gathered}
$$

Therefore $\mathbf{U}^{\varepsilon}$ is bounded in $\operatorname{SBD}\left(\Sigma^{1}\right)$ and $\mathcal{H}^{2}\left(J_{\mathbf{U}^{\varepsilon}}\right)$ is bounded too. Hence $\mathbf{U}^{\varepsilon} \rightarrow \mathbf{U}=\left(\mathbf{u}, u_{3}\right)$ in $w^{*} S B D$; moreover (5.10) implies $\nabla_{t} u_{3}=0$ a.e. in $\Sigma^{1}$ while (5.8) and Corollary 1.3 of [9] entail

$$
\int_{J_{\mathbf{U}}}\left|\left[u_{3}\right]\left(\nu_{\mathbf{U}}\right)_{3}\right| \mathrm{d} \mathcal{H}^{2} \leqslant \liminf _{\varepsilon \rightarrow 0} \int_{J_{\mathbf{U}^{\varepsilon}}}\left|\left[u_{3}^{\varepsilon}\right]\left(\nu_{\mathbf{U}^{\varepsilon}}\right)_{3}\right| \mathrm{d} \mathcal{H}^{2}=0 .
$$

Then spt $u_{3} \subset \bar{\Omega}$ and

$$
D_{3} u_{3}=\mathcal{E}_{33}(\mathbf{U}) \mathrm{d} \mathbf{x} \mathrm{d} t+\left[u_{3}\right]\left(\nu_{\mathbf{U}}\right)_{3} \mathrm{~d} \mathcal{H}^{2}(\mathbf{x}, t)\left\llcorner J_{\mathbf{U}}=0\right.
$$

hence $u_{3}$ does not depend on $z$, and by taking into account (5.11),

$$
\left[u_{3}\right]\left(\nu_{\mathbf{U}}\right)_{3}=\left[u_{3}\right]=\left(\nu_{\mathbf{U}^{\varepsilon}}\right)_{3}=0 .
$$

By using (5.9) and (5.11) it is easy to see that $\mathcal{E}_{\mathbf{x}}\left(\mathbf{U}^{\varepsilon}\right)$, is bounded in $L^{2}\left(\Sigma^{1}\right)$, for $\alpha, \beta=1,2$, $\mathcal{E}_{\alpha, \beta}\left(\mathbf{U}^{\varepsilon}\right) \rightarrow \mathcal{E}_{\alpha, \beta}(\mathbf{U})$ weakly in $L^{2}$, and $\mathrm{e}_{\alpha 3}\left(\mathbf{U}^{\varepsilon}\right) \rightarrow 0$ in the strong topology of measures and then for every $\varphi \in C_{0}^{0}(\Omega)$ we have

$$
\left\langle\mathbf{U}_{\alpha, 3}^{\varepsilon}+\mathbf{U}_{3, \alpha}^{\varepsilon}, \varphi\right\rangle=-\int_{\Sigma^{1}}\left(\mathbf{U}_{\alpha}^{\varepsilon} \varphi_{, 3}+u_{3}^{\varepsilon} \varphi_{, \alpha}\right) \mathrm{d} \mathbf{x} \mathrm{d} t \rightarrow-\int_{\Sigma^{1}}\left(\mathbf{U}_{\alpha} \varphi_{, 3}+u_{3} \varphi_{, \alpha}\right) \mathrm{d} \mathbf{x} \mathrm{d} t=0
$$

say $\mathrm{e}_{\alpha 3}(\mathbf{U})=0$. Then, by $\mathbf{U}_{3}=u_{3}(\mathbf{x})$, we get

$$
\mathbf{u}(\mathbf{x}, t)=\zeta(\mathbf{x})-t D u_{3}(\mathbf{x}) .
$$

So far we have proved that $E^{0}(\mathbf{U})<+\infty$ if and only if $\mathbf{U} \in \mathcal{A}$. Now, by (3.7), (5.3), we have

$$
\begin{gathered}
\varepsilon^{-2} \int_{J_{\mathbf{U}^{\varepsilon}}} \theta^{\varepsilon}\left(\varepsilon\left[\mathbf{u}^{\varepsilon}\right],\left[u_{3}^{\varepsilon}\right], \overline{\nu_{\mathbf{U}^{\varepsilon}}}, \varepsilon^{-1}\left(\nu_{\mathbf{U}^{\varepsilon}}\right)_{3}\right) \mathrm{d} \mathcal{H}^{2} \geqslant \delta \mathcal{H}^{2}\left(J_{\mathbf{U}^{\varepsilon}}\right)+\gamma \int_{J_{\mathbf{U}^{\varepsilon}}}\left|\left[\mathbf{U}^{\varepsilon}\right] \odot v\left(\mathbf{U}^{\varepsilon}\right)\right| \mathrm{d} \mathcal{H}^{2} \\
\mu\left|\mathcal{E}_{\mathbf{x}}\left(\mathbf{U}^{\varepsilon}\right)\right|^{2}+\frac{\lambda \mu}{\lambda+2 \mu}\left|\operatorname{Tr} \mathcal{E}_{\mathbf{x}}\left(\mathbf{U}^{\varepsilon}\right)\right|^{2}=\varepsilon^{-2} \min \left(\mu\left|\widetilde{\mathcal{E}}\left(\mathbf{U}^{\varepsilon}\right)\right|^{2}+\frac{\lambda}{2}\left|\operatorname{Tr}\left(\widetilde{\mathcal{E}}\left(\mathbf{U}^{\varepsilon}\right)\right)\right|^{2}\right)
\end{gathered}
$$


where the minimum is taken over the third row and column of the matrix $\widetilde{\mathcal{E}}\left(\mathbf{U}^{\varepsilon}\right)$. Then, by (3.11), (5.4),

$$
\begin{aligned}
& E^{\mathcal{E}}\left(\mathbf{U}^{\varepsilon}\right)=\varepsilon^{-2} \int_{\Sigma^{1}}\left(\mu\left|\widetilde{\mathcal{E}}\left(\mathbf{U}^{\varepsilon}\right)\right|^{2}+\frac{\lambda}{2}\left|\operatorname{Tr}\left(\widetilde{\mathcal{E}}\left(\mathbf{U}^{\varepsilon}\right)\right)\right|^{2}\right) \mathrm{d} \mathbf{x} \mathrm{d} t \\
& +\varepsilon^{-2} \int_{J_{\mathbf{U}^{\varepsilon}}} \theta^{\varepsilon}\left(\varepsilon\left[\mathbf{u}^{\varepsilon}\right],\left[u_{3}^{\varepsilon}\right], \overline{\nu_{\mathbf{U}^{\varepsilon}}}, \varepsilon^{-1}\left(\nu_{\mathbf{U}^{\varepsilon}}\right)_{3}\right) \mathrm{d} \mathcal{H}^{2}-\frac{1}{2} \int_{\Sigma^{1}}\left(g u_{3}^{\varepsilon}+\varepsilon \mathbf{g} \cdot \mathbf{u}^{\varepsilon}\right) \mathrm{d} \mathbf{x} \mathrm{d} t \\
& \geqslant \int_{\Sigma^{1}}\left(\mu\left|\mathcal{E}_{\mathbf{x}}\left(\mathbf{U}^{\varepsilon}\right)\right|^{2}+\frac{\lambda \mu}{\lambda+2 \mu}\left|\operatorname{Tr} \mathcal{E}_{\mathbf{x}}\left(\mathbf{U}^{\varepsilon}\right)\right|^{2}\right) \mathrm{d} \mathbf{x} \mathrm{d} t \\
& +\delta \mathcal{H}^{2}\left(J_{\mathbf{U}^{\varepsilon}}\right)+\gamma \int_{J_{\mathbf{U}^{\varepsilon}}}\left|\left[\mathbf{U}^{\varepsilon}\right] \odot \nu_{\mathbf{U}^{\varepsilon}}\right| \mathrm{d} \mathcal{H}^{2}-\frac{1}{2} \int_{\Sigma^{1}}\left(g u_{3}^{\varepsilon}+\varepsilon \mathbf{g} \cdot \mathbf{u}^{\varepsilon}\right) \mathrm{d} \mathbf{x} \mathrm{d} t \\
& \geqslant \int_{\Sigma}\left\{2 \mu\left|\mathcal{E}_{\mathbf{x}}(\zeta)\right|^{2}+\frac{2 \lambda \mu}{\lambda+2 \mu}|\operatorname{div} \zeta|^{2}+\frac{2}{3}\left(\mu\left|\nabla^{2} u_{3}\right|^{2}+\frac{\lambda \mu}{\lambda+2 \mu}\left|\Delta^{a} u_{3}\right|^{2}\right)\right\} \mathrm{d} \mathbf{x} \\
& +\int_{\Sigma^{1}}\left\{2 \mu\left(\mathcal{E}_{\mathbf{x}}(\boldsymbol{\zeta})-t \nabla^{2} u_{3}\right)+\frac{2 \lambda \mu}{\lambda+2 \mu}\left(\operatorname{div} \zeta-t \Delta^{a} u_{3}\right) I_{2}\right\}: \mathcal{E}_{\mathbf{x}}\left(\mathbf{U}^{\varepsilon}-\zeta+t D u_{3}\right) \mathrm{d} \mathbf{x} \mathrm{d} t \\
& +\delta \mathcal{H}^{2}\left(J_{\mathbf{U}^{\varepsilon}}\right)+\gamma \int_{J_{\mathbf{U}^{\varepsilon}}}\left|\left[\mathbf{U}^{\varepsilon}\right] \odot \nu_{\mathbf{U}^{\varepsilon}}\right| \mathrm{d} \mathcal{H}^{2}-\frac{1}{2} \int_{\Sigma^{1}}\left(g u_{3}^{\varepsilon}+\varepsilon \mathbf{g} \cdot \mathbf{u}^{\varepsilon}\right) \mathrm{d} \mathbf{x} \mathrm{d} t .
\end{aligned}
$$

In the second inequality we exploited cancellation of odd terms in $t$ and the following inequality: $\varphi(A) \geqslant \varphi(B)+D \varphi(B):(A-B))$ for $C^{1}$, convex $\varphi: M_{2,2} \rightarrow \mathbb{R}$, with $\varphi(A)=\mu|A|^{2}+$ $\frac{\lambda \mu}{\lambda+2 \mu}|\operatorname{Tr} A|^{2}, A=\mathcal{E}_{\mathbf{x}}\left(\mathbf{U}^{\varepsilon}\right)$ and $B=\mathcal{E}_{\mathbf{x}}\left(\zeta-t D u_{3}\right)=\mathcal{E}_{\mathbf{x}}(\zeta)-t \nabla^{2} u_{3}$. Taking into account (5.15) and (5.16) we get

$$
\int_{\Sigma^{1}}\left\{2 \mu\left(\mathcal{E}_{\mathbf{x}}(\zeta)-t \nabla^{2} u_{3}\right)+\frac{2 \lambda \mu}{\lambda+2 \mu}\left(\operatorname{div} \zeta-t \Delta u_{3}\right) I_{2}\right\}: \mathcal{E}_{\mathbf{x}}\left(\mathbf{U}^{\varepsilon}-\zeta+t D u_{3}\right) \mathrm{d} \mathbf{x} \mathrm{d} t \rightarrow 0
$$

and by lower semi-continuity we get

$$
\liminf _{\varepsilon \rightarrow 0}\left(\delta \mathcal{H}^{2}\left(J_{\mathbf{U}^{\varepsilon}}\right)+\gamma \int_{J_{\mathbf{U}^{\varepsilon}}}\left|\left[\mathbf{U}^{\varepsilon}\right] \odot v\left(\mathbf{U}^{\varepsilon}\right)\right| \mathrm{d} \mathcal{H}^{2}\right) \geqslant \delta \mathcal{H}^{2}\left(J_{\mathbf{U}}\right)+\gamma \int_{J_{\mathbf{U}}}|[\mathbf{U}] \odot v(\mathbf{U})| \mathrm{d} \mathcal{H}^{2} .
$$

Moreover

$$
\frac{1}{2} \int_{\Sigma^{1}}\left(g u_{3}^{\varepsilon} \mathrm{d} \mathbf{x} \mathrm{d} t+\varepsilon \mathbf{g} \cdot \mathbf{u}^{\varepsilon}\right) \mathrm{d} x \mathrm{~d} y \mathrm{~d} t \rightarrow \int_{\Omega} g u_{3} \mathrm{~d} x \mathrm{~d} y
$$

and gathering together (5.17), (5.18) and (5.19) we obtain that

$$
\liminf _{\varepsilon \rightarrow 0} E^{\varepsilon}\left(\mathbf{U}^{\varepsilon}\right) \geqslant E^{0}(\mathbf{U}) \text {. }
$$

Proof of Theorem 3.4-Step 2: upper bound for the $\Gamma$ limit.

Since $\Delta^{a} u_{3}$, div $\zeta$ belong to $L^{2}(\Sigma)$ we can choose $\mathbf{U} \in \mathcal{A}$, that is $\mathbf{U}(\mathbf{x}, t)=\left(\boldsymbol{\zeta}(\mathbf{x})-t D u_{3}(\mathbf{x})\right.$, $\left.u_{3}(\mathbf{x})\right)$, and, for every $k>0$, two smooth scalar functions $\varphi_{k}, \psi_{k}$ defined on $\Sigma$ such that

$$
\begin{aligned}
& \left\|\varphi_{k}+\frac{\lambda}{\lambda+2 \mu} \Delta^{a} u_{3}\right\|_{L^{2}(\Sigma)} \leqslant \frac{1}{k} \\
& \left\|\psi_{k}+\frac{\lambda}{\lambda+2 \mu} \operatorname{div} \zeta\right\|_{L^{2}(\Sigma)} \leqslant \frac{1}{k} .
\end{aligned}
$$


We define now the recovery sequences

$$
\begin{aligned}
& \mathbf{U}_{k}^{\varepsilon}(\mathbf{x}, t)=\left(\zeta(\mathbf{x})-t \nabla u_{3}(\mathbf{x}), u_{3}(\mathbf{x})+\varepsilon^{2} t \psi_{k}(\mathbf{x})-\frac{1}{2}(\varepsilon t)^{2} \varphi_{k}(\mathbf{x})\right) \\
& \mathbf{V}^{\varepsilon}(\mathbf{x}, z)=\left(\mathbf{v}^{\varepsilon}(\mathbf{x}, z), v_{3}^{\varepsilon}(\mathbf{x}, z)\right) \quad \text { where } \quad \mathbf{v}^{\varepsilon}(\mathbf{x}, z)=\varepsilon \mathbf{u}^{\varepsilon}(\mathbf{x}, z / \varepsilon), v_{3}^{\varepsilon}(\mathbf{x}, z)=u_{3}^{\varepsilon}(\mathbf{x}, z / \varepsilon),
\end{aligned}
$$

then

$$
\mathbf{U}_{k}^{\varepsilon} \stackrel{L^{1}\left(\Sigma^{1}, \mathbb{R}^{3}\right)}{\longrightarrow} \mathbf{U}=\left(\mathbf{u}, u_{3}\right) \quad \text { as } \varepsilon \rightarrow 0_{+}, \text {for every } k>0 .
$$

Moreover $\left(v_{\mathbf{U}_{k}^{\varepsilon}}^{\varepsilon}\right)_{3}=0,\left[\mathbf{U}_{k, 3}^{\varepsilon}\right]=0$ and, referring to (5.4), (3.11),

$$
\begin{aligned}
\tilde{\mathcal{E}}\left(\mathbf{U}_{k}^{\varepsilon}\right)=\left(\begin{array}{cc}
\varepsilon \mathcal{E}_{\mathbf{x}}\left(\zeta-t \nabla u_{3}\right) & \frac{\varepsilon^{2} t}{2}\left(\nabla \psi_{k}-\frac{t}{2} \nabla \varphi_{k}\right) \\
\frac{\varepsilon^{2} t}{2}\left(\nabla \psi_{k}-\frac{t}{2} \nabla \varphi_{k}\right) & \varepsilon^{-1}\left(\varepsilon^{2} \psi_{k}-t \varepsilon^{2} \varphi_{k}\right)
\end{array}\right) \\
E^{\varepsilon}\left(\mathbf{U}_{k}^{\varepsilon}\right)=\int_{\Sigma} 2\left\{\left(\mu\left|\mathcal{E}_{\mathbf{x}}(\zeta)\right|^{2}+\mu\left|\psi_{k}\right|^{2}+\frac{\lambda}{2}\left|\operatorname{div} \zeta+\psi_{k}\right|^{2}\right)\right. \\
\left.+\frac{2}{3}\left(\mu\left|\nabla^{2} u_{3}\right|^{2}+\mu\left|\varphi_{k}\right|^{2}+\frac{\lambda}{2}\left|\Delta^{a} u_{3}+\varphi_{k}\right|^{2}\right)+\frac{\varepsilon^{2}}{3}\left|\nabla \psi_{k}\right|^{2}+\frac{\varepsilon^{2}}{5}\left|\nabla \varphi_{k}\right|^{2}\right\} \mathrm{d} x \mathrm{~d} y \\
+\int_{\mathbf{J}_{k}^{\varepsilon}}\left(\delta+\gamma\left|\left[\mathbf{U}_{k}^{\varepsilon}\right] \odot v\left(\mathbf{U}_{k}^{\varepsilon}\right)\right|\right) \mathrm{d} \mathcal{H}^{2}(\mathbf{x}, t)-\int_{\Sigma}\left(g\left(u_{k}^{\varepsilon}\right)_{3}+\varepsilon \mathbf{g} \cdot \mathbf{u}_{k}^{\varepsilon}\right) \mathrm{d} \mathbf{x} \mathrm{d} t .
\end{aligned}
$$

By (5.21) and (5.22) it is easy to see that there exists a constant $C$ dependent of $\lambda, \mu$ but independent of $\varepsilon$ such that, for every $k>0$,

$$
\begin{aligned}
& \limsup _{\varepsilon \rightarrow 0} E^{\varepsilon}\left(\mathbf{U}_{k}^{\varepsilon}\right) \\
& \leqslant \int_{\Sigma}\left\{\frac{2}{3} \mu\left(\left|\nabla^{2} u_{3}\right|^{2}+\frac{\lambda}{\lambda+2 \mu}\left|\Delta^{a} u_{3}\right|^{2}\right)+2 \mu\left(\left|\mathcal{E}_{\mathbf{x}}(\zeta)\right|^{2}+\frac{\lambda}{\lambda+2 \mu}|\operatorname{div} \zeta|^{2}\right)\right\} \mathrm{d} x \mathrm{~d} y \\
& \quad+c k^{-2}+\int_{J_{\mathbf{U}}}(\delta+\gamma|[\mathbf{U}] \odot v(\mathbf{U})|) \mathrm{d} \mathcal{H}^{2}(x, y, t)-\int_{\Sigma} g u_{3} \mathrm{~d} x \mathrm{~d} y=E^{0}(\mathbf{U})+c k^{-2} .
\end{aligned}
$$

Then by a standard diagonal procedure there exists $\mathbf{U}_{k^{\varepsilon}}^{\varepsilon}$ such that $\mathbf{U}_{k^{\varepsilon}}^{\varepsilon} \rightarrow \mathbf{U}$ in $L^{1}\left(\Sigma^{1}\right)$ and

$$
\limsup _{\varepsilon \rightarrow 0} E^{\varepsilon}\left(\mathbf{U}_{k^{\varepsilon}}^{\varepsilon}\right) \leqslant E^{0}(\mathbf{U})
$$

Theorem 3.4 is proved by summarizing (5.20), (5.26) and Definition 3.2.

Proof of Theorem 3.6-Convergence of minimizers.

Let $\mathbf{V}^{\varepsilon}=\left(\mathbf{v}^{\varepsilon}, v_{3}^{\varepsilon}\right) \in \operatorname{argmin}\left(\mathbf{L} \mathbf{P}^{\varepsilon}\right)$ and $\mathbf{U}^{\varepsilon}(x, t)=\left(\varepsilon^{-1} \mathbf{v}^{\varepsilon}(\mathbf{x}, \varepsilon t), v_{3}^{\varepsilon}(\mathbf{x}, \varepsilon t)\right)$, then $\mathbf{U}^{\varepsilon}$ minimizes $E^{\varepsilon}$ and

$$
E^{\varepsilon}\left(\mathbf{U}^{\varepsilon}\right) \leqslant E^{\varepsilon}(\mathbf{0})=0 .
$$


By arguing as in the proof of Theorem 3.4, we find that (5.6)-(5.15) hold in the present case too, that there exist $\boldsymbol{\xi} \in \operatorname{SBD}(\Sigma), \omega \in \operatorname{SBH}(\Sigma)$ with support contained in $\bar{\Omega}$ such that, up to subsequences,

$$
\left(\mathbf{u}^{\varepsilon}, u_{3}^{\varepsilon}\right) \stackrel{w^{*} S B D\left(\Sigma^{1}\right)}{\longrightarrow}(\boldsymbol{\xi}-t D \omega, \omega)=\mathbf{W}
$$

and that, by Theorems 3.3, 3.4, W minimizes $E^{0}(\mathbf{U})$.

We claim that $\boldsymbol{\xi}=\mathbf{0}$ : the first step is proving that $\mathcal{E}_{\mathbf{x}}(\boldsymbol{\xi})=\mathbf{0} \otimes \mathbf{0}$. Indeed if $\mathcal{E}_{\mathbf{x}}(\boldsymbol{\xi}) \not \equiv \mathbf{0} \otimes \mathbf{0}$ then

$$
\int_{\Omega} \mu\left(\left|\mathcal{E}_{\mathbf{x}}(\boldsymbol{\xi})\right|^{2}+\frac{\lambda}{\lambda+2 \mu}\left|\operatorname{Tr} \mathcal{E}_{\mathbf{x}}(\boldsymbol{\xi})\right|^{2}\right) \mathrm{d} \mathbf{x}>0
$$

hence by Lemma 2.2 (with $\varepsilon=1, \tau=1$ )

$$
E^{0}(\mathbf{W})>E^{0}(-t D \omega, \omega)
$$

which contradicts the minimality of $\mathbf{W}$. We show now that $J_{\boldsymbol{\xi}}=\emptyset$ : if this identity were false, by (5.14), $v_{3}(\mathbf{W})=0$, hence ([30: Theorem 3.2.19])

$$
\begin{gathered}
v(\mathbf{W})=(\bar{v}(D \omega), 0) \quad \text { on } J_{D \omega} \backslash J_{\xi} \text { if } t \neq 0 \\
v(\mathbf{W})=(\bar{v}(\boldsymbol{\xi}), 0)=(\bar{v}(D \omega), 0) \quad \mathcal{H}^{1} \text { a.e. } J_{\boldsymbol{\xi}} \cap J_{D \omega} \text { except at most one single value of } t,
\end{gathered}
$$

then Lemma 2.3 and Lemma 2.2 with $\tau=\varepsilon=1$ yield,

$$
\begin{aligned}
& E^{0}(\mathbf{W})+\int_{\Sigma} g \omega \mathrm{d} \mathbf{x} \\
= & \frac{2}{3} \mu \int_{\Sigma}\left(\frac{\lambda}{\lambda+2 \mu}\left|\Delta^{a} \omega\right|^{2}+\left|\nabla^{2} \omega\right|^{2}\right) \mathrm{d} \mathbf{x}+\int_{J_{\mathbf{W}}}(\delta+\gamma|[\mathbf{W}] \odot v(\mathbf{W})|) \mathrm{d} \mathcal{H}^{2}(\mathbf{x}, t) \\
\geqslant & \frac{2}{3} \mu \int_{\Sigma}\left(\frac{\lambda}{\lambda+2 \mu}\left|\Delta^{a} w\right|^{2}+\left|\nabla^{2} w\right|^{2}\right) \mathrm{d} \mathbf{x} \\
& +\int_{-1}^{1}\left(\int_{J_{\boldsymbol{\xi}} \cap J_{D \omega}}(\delta+\gamma|[\boldsymbol{\xi}-t D \omega] \odot \bar{v}(\boldsymbol{\xi}-t D \omega)|) \mathrm{d} \mathcal{H}^{1}(\mathbf{x})\right) \mathrm{d} t \\
& +\int_{-1}^{1}\left(\int_{J_{D \omega} \backslash J_{\xi}}(\delta+\gamma|[\xi-t D \omega] \odot \bar{\nu}(D \omega)|) \mathrm{d} \mathcal{H}^{1}(\mathbf{x})\right) \mathrm{d} t \\
> & \frac{2}{3} \mu \int_{\Sigma}\left(\left|\nabla^{2} \omega\right|^{2}+\frac{\lambda}{\lambda+2 \mu}\left|\Delta^{a} \omega\right|^{2}\right) \mathrm{d} \mathbf{x} \\
& +\int_{J_{\xi} \cap J_{D \omega}}(2 \delta+\gamma|[\nabla \omega] \odot \bar{\nu}(D \omega)|) \mathrm{d} \mathcal{H}^{1}(\mathbf{x})+\int_{J_{D \omega} \backslash J_{\xi}}(2 \delta+\gamma|[D \omega] \odot \bar{v}(D \omega)|) \mathrm{d} \mathcal{H}^{1}(\mathbf{x}) \\
= & E^{0}(-t D \omega, \omega)+\int_{\Sigma} g \omega \mathrm{d} \mathbf{x}
\end{aligned}
$$

which gives a contradiction too. Therefore $\boldsymbol{\xi}$ is a rigid plane displacement field in $\Sigma$. Recalling now that spt $\boldsymbol{\xi} \subset \bar{\Omega}$ we have that $\boldsymbol{\xi}=\mathbf{0}$ in $\Sigma$ and the proof of Theorem 3.6 is achieved.

Proof of 4.2-Approximation of the rigid plastic plate. 
The proofs of Theorems 3.4 and 3.6 may be easily adapted in order to obtain Theorem 4.2. Indeed by repeating the same arguments of estimates (5.6) and (5.7) we have that inequalities (5.8)(5.12) continue to hold whenever $\mathbf{V}^{\varepsilon} \in \operatorname{argmin}\left(\Lambda^{\varepsilon}\right)$. Moreover, having in mind formulae (4.3), we have

$$
\int_{\Sigma^{1}}\left|\widetilde{\mathcal{E}}\left(\mathbf{U}^{\varepsilon}\right)\right|^{2} \mathrm{~d} \mathbf{x} \mathrm{d} t \leqslant c \varepsilon^{3}
$$

and therefore the technique of the proof of Theorem 3.4 permits to show that the $\Gamma$-limit $\mathcal{W}^{0}$ is finite if and only if $\mathbf{U} \in \mathcal{A}$. More precisely, (5.27) yields that $\mathcal{E}_{\mathbf{x}}(\mathbf{U})=\mathcal{E}_{\mathbf{x}}(\zeta)-t \nabla^{2} u_{3}=\mathbf{0} \otimes \mathbf{0}$ for a.e. $t \in(-1,1)$, then $\mathcal{E}_{\mathbf{x}}(\zeta)=0=\nabla^{2} u_{3}$ a.e. in $\Sigma, \mathcal{T}^{0}(\mathbf{U})<+\infty$ iff $\mathbf{U} \in \mathcal{U}$ and $\mathcal{W}^{0}$ has the explicit representation claimed in Theorem 4.2. In order to conclude the proof it is enough to take into account (5.27) and to repeat the arguments of the proofs of Theorem 3.6.

\section{Some remarks about the Kirchhoff cinematic restriction}

In this section we assume the usual Kirchhoff cinematic restriction on the deformations of thin bodies, namely 'the material fibres orthogonal to the middle surface before loading remain approximately orthogonal to it after loading and suffer negligible stretching' [33]. Kirchhoff restriction can be written in the following way for linearly elastic bodies (see [39]): e(V) $\cdot \mathbf{n}=\mathbf{0}$ where $\mathbf{n}$ denotes the prescribed normal to the middle line (or surface). Explicitly, by assuming (3.1)-(3.3), the cinematic restriction is

$$
\mathrm{e}(\mathbf{V}) \cdot \mathbf{e}_{3} \equiv \mathbf{0} \quad \text { in } \mathcal{D}^{\prime}\left(\Sigma^{\varepsilon}, \mathbb{R}^{3}\right) .
$$

This hypothesis entails a precise (and simpler) geometric structure of admissible deformations and fractures, as shown by the following lemma, whose statement is well known and easily achievable for smooth or Sobolev displacements-but it is proved here for $S B D$ functions.

Lemma 6.1 Let $m=3, \mathbf{V} \in \operatorname{SBD}\left(\Sigma^{\varepsilon}\right), \mathbf{V}=\left(\mathbf{v}, v_{3}\right), \mathbf{v}=\left(v_{1}, v_{2}\right)$ such that $\mathrm{e}(\mathbf{V}) \cdot \mathbf{e}_{3}=\mathbf{0}$ in the sense of measures. Then $v_{3}=v_{3}(x, y) \in \operatorname{SBH}(\Sigma)$ and there exists $\zeta \in S B D(\Sigma)$ such that

$$
\begin{gathered}
\mathbf{v}=\zeta-z D v_{3} . \\
J_{\mathbf{V}}=\left(J_{\zeta} \cup S_{D v_{3}}\right) \times(-\varepsilon, \varepsilon) \quad \text { up to a set of null } \mathcal{H}^{2} \text { measure. }
\end{gathered}
$$

Moreover $\mathrm{e}_{\mathbf{x}}(\mathbf{V})=\mathrm{e}_{\mathbf{x}}(\mathbf{v})-z D^{2} v_{3}$ say, denoting by commas the distributional partial derivatives, $v_{1}=\zeta_{1}-z v_{3, x}, v_{2}=\zeta_{2}-z v_{3, y}$, and

$$
\begin{gathered}
\mathrm{e}(\mathbf{V})=\left(\begin{array}{ccc}
\zeta_{1, x}-z v_{3, x x} & \frac{1}{2}\left(\zeta_{1, y}+\zeta_{2, x}\right)-z v_{3, x y} & 0 \\
\frac{1}{2}\left(\zeta_{1, y}+\zeta_{2, x}\right)-z v_{3, x y} & \zeta_{2, y}-z v_{3, y y} & 0 \\
0 & 0 & 0
\end{array}\right) \\
\mathcal{E}_{\mathbf{x}}(\mathbf{V})=\mathcal{E}(\mathbf{v})=\mathcal{E}(\zeta)-z \nabla^{2} v_{3} \\
\operatorname{div} \mathbf{V}=\operatorname{Tr} \mathrm{e}^{a}(\mathbf{V})=\operatorname{Tr} \mathcal{E}_{\mathbf{x}}(\mathbf{V})=\operatorname{div} \zeta-z \Delta^{a} v_{3} .
\end{gathered}
$$

Proof. Since $\mathbf{V} \in \operatorname{SBD}\left(\Sigma^{\varepsilon}\right)$ and $\mathrm{e}(\mathbf{V}) \cdot \mathbf{e}_{3}=\mathbf{0}$ in the sense of measures we get that $D_{z} v_{3}=0$, $v_{1, z}=-v_{3, x}, v_{2, z}=v_{3, y}$ and

$$
\left\{\begin{array}{l}
\mathbf{v}=\zeta-z D v_{3} \\
v_{3}=v_{3}(x, y)
\end{array}\right.
$$


with $\zeta, v_{3} \in \mathcal{D}^{\prime}(\Sigma)$. Since $\mathbf{V} \in \operatorname{SBD}\left(\Sigma^{\varepsilon}\right)$, Theorem 4.5 and Proposition 4.7 of [3] entail $\mathbf{v}(\cdot, \cdot, z) \in$ $S B D(\Sigma)$ for a.e. $z \in(-\varepsilon, \varepsilon)$ therefore $\zeta, D v_{3}, \in S B D(\Sigma)$ by a standard elimination procedure and (6.2) holds a.e. in $\Sigma^{\varepsilon}$. By e $\left(D v_{3}\right)=D^{2} v_{3}$ we get $v_{3} \in S B H$. Then $\left(v_{\mathbf{V}}\right)_{3}=0$ and $J_{\mathbf{V}} \subset$ $\left(J_{\zeta} \cup J_{D v_{3}}\right) \times(-\varepsilon, \varepsilon)$ : the two sets may differ only where there is cancellation (allowed, for $\mathcal{H}^{1}$ a.e. $x, y$, for at most one $z$ ) then (6.3) is proven. (6.4)-(6.6) follow by differentiating (6.2) and this concludes the proof.

Let now $\mathbf{F}^{\varepsilon}, \mathbf{G}, \mathcal{G}^{\varepsilon}, \mathcal{F}^{\varepsilon}, \mathcal{L}^{\varepsilon}$ as in (3.4)-(3.9); we consider here the following Dirichlet problem subject to the Kirchhoff cinematic constraint:

$$
\min \left\{\mathcal{F}^{\varepsilon}(\mathbf{V}): \mathbf{V} \in \operatorname{SBD}\left(\Sigma^{\varepsilon}\right), \text { s.t. } \operatorname{spt} \mathbf{V} \subset \overline{\Omega^{\varepsilon}}, \mathrm{e}(\mathbf{V}) \cdot \mathbf{e}_{3} \equiv \mathbf{0}\right\}
$$

$\left(\mathbf{K L P}^{\varepsilon}\right)$

The same techniques of Section 5 together with (6.1)-(6.6) show

$$
\begin{aligned}
& \int_{\Sigma^{\varepsilon}}\left(\mu|\mathcal{E}(\mathbf{V})|^{2}+\frac{\lambda}{2}|\operatorname{Tr} \mathcal{E}(\mathbf{V})|^{2}\right) \mathrm{d} \mathbf{X} \\
& \quad=\varepsilon^{2} \frac{2}{3} \mu \int_{\Sigma}\left(\left|\nabla^{2} v_{3}\right|^{2}+\frac{\lambda}{2 \mu}\left|\Delta^{a} v_{3}\right|^{2}\right) \mathrm{d} \mathbf{x}+\varepsilon 2 \mu \int_{\Sigma}\left(|\mathcal{E}(\zeta)|^{2}+\frac{\lambda}{2 \mu}(\operatorname{div} \zeta)^{2}\right) \mathrm{d} \mathbf{x}
\end{aligned}
$$

and hence the following statement.

Theorem 6.2 Assume that $m=3,(3.1)-(3.10)$ and (6.1) are satisfied. Then, for every $\varepsilon>0$, $\left(\mathbf{K L} \mathbf{P}^{\varepsilon}\right)$ admits a solution $\mathbf{V}^{\varepsilon} \in \operatorname{SBD}\left(\Sigma^{\varepsilon}\right), \mathbf{V}^{\varepsilon}(x, z)=\left(\zeta^{\varepsilon}-z \nabla v_{3}^{\varepsilon}, v_{3}^{\varepsilon}\right)$ such that spt $\mathbf{V}^{\varepsilon} \subset \bar{\Omega}^{\varepsilon}$

$$
\varepsilon^{-1} \zeta^{\varepsilon} \stackrel{w^{*}-S B D(\Sigma)}{\longrightarrow} \mathbf{0}, \quad v_{3}^{\varepsilon} \stackrel{w^{*}-S B H(\Sigma)}{\longrightarrow} v_{3}
$$

where $v_{3}$ is a suitable minimizer of

$\mathcal{K}(w)=\frac{2}{3} \mu \int_{\Sigma}\left(\left|\nabla^{2} w\right|^{2}+\frac{\lambda}{2 \mu}\left|\Delta^{a} w\right|^{2}\right) \mathrm{d} x \mathrm{~d} y+2 \delta \mathcal{H}^{1}\left(S_{D w}\right)+\gamma \int_{S_{D w}}|[D w]| \mathrm{d} \mathcal{H}^{1}-\int_{\Sigma} g w \mathrm{~d} x \mathrm{~d} y$

among all $w \in \operatorname{SBH}(\Sigma)$ such that $w=0$ on $\Sigma \backslash \Omega$.

REMARK 6.3 It is worth noticing that for every competing function $w$

$$
\mathcal{G}^{0}(w) \leqslant \mathcal{K}(w)
$$

and the equality occurs if and only if either $\lambda=0$ or $\Delta^{a} w \equiv 0$. A direct calculation allows us to show that in the elastic framework a strict inequality holds also for minimizers of $\mathcal{G}^{0}$ and $\mathcal{K}$ respectively. Therefore we can say that when $\lambda>0$, even if free gradient discontinuities develop, one may have

$$
\min \mathcal{G}^{0}<\min \mathcal{K}
$$

for all loads and shapes such that at least one minimizer $u_{0}$ of $\mathcal{K}$ fulfills $\Delta^{a} u_{0} \not \equiv 0$. Actually, such a case may happen: explicit examples can be shown for the elastic plastic beam.

Finally we show an analytical deduction of a quantitative estimate for Griffith critical length from safe load condition and Kirchhoff assumption: the estimate depends explicitly of the geometry of the plate and the Lamé coefficients of the material and is dimensionally consistent. 
THEOREM 6.4 If $n=3$ and (3.1)-(3.9) hold, then the safe load (3.10) entails an estimate on the area of crack for minimizers $\mathbf{V}^{\varepsilon}$ of problem $\left(\mathbf{L} \mathbf{P}^{\varepsilon}\right)$

$$
\mathcal{H}^{2}\left(J_{\mathbf{V}^{\varepsilon}}\right)<6 \frac{\gamma^{2}}{\mu \delta}|\Sigma| \varepsilon
$$

If Kirchhoff cinematic restriction (6.1) holds too, then the following estimate of the Griffith critical length follows:

$$
\mathcal{H}^{1}\left(J_{\boldsymbol{\zeta}^{\varepsilon}} \cup S_{D v_{3}^{\varepsilon}}\right)<3 \frac{\gamma^{2}}{\mu \delta}|\Sigma| .
$$

Proof. From $p \geqslant n=3,2 \mu+3 \lambda>0,|\Omega|<|\Sigma|, \mathcal{F}^{\varepsilon}\left(\mathbf{V}^{\varepsilon}\right) \leqslant \mathcal{F}^{\varepsilon}\left(\mathbf{0}^{\varepsilon}\right)=0$, we get

$$
\begin{aligned}
& \frac{\mu}{3} \int_{\Sigma^{\varepsilon}}\left|\mathcal{E}\left(\mathbf{V}^{\varepsilon}\right)\right|^{2} \mathrm{~d} \mathbf{X}+\gamma \varepsilon \int_{J_{\mathbf{V}^{\varepsilon}}} \mid\left[\mathbf{V}^{\varepsilon} \odot v\left(\mathbf{V}^{\varepsilon}\right] \mid \mathrm{d} \mathcal{H}^{2}(\mathbf{X})+\varepsilon^{2} \delta \mathcal{H}^{2}\left(J_{\mathbf{V}^{\varepsilon}}\right)\right. \\
& \leqslant \mathcal{G}^{\varepsilon}\left(\mathbf{V}^{\varepsilon}\right) \leqslant \mathcal{L}^{\varepsilon}\left(\mathbf{V}^{\varepsilon}\right) \leqslant \frac{\varepsilon^{2}}{2} \int_{\Sigma^{\varepsilon}} \mathbf{G} \cdot \mathbf{V}^{\varepsilon} \mathrm{d} x \mathrm{~d} y \leqslant 2^{(1-p) / p} \varepsilon^{2+1 / p}\|\mathbf{G}\|_{L^{p}(\Omega)}\left\|\mathbf{V}^{\varepsilon}\right\|_{L^{p^{\prime}}\left(\Sigma^{\varepsilon}\right)} \\
& \stackrel{\text { safe load }}{<\varepsilon}\left\{\int_{\Sigma^{\varepsilon}}\left|\mathcal{E}\left(\mathbf{V}^{\varepsilon}\right)\right| \mathrm{d} \mathbf{X}+\int_{J_{\mathbf{V}^{\varepsilon}}} \mid\left[\mathbf{V}^{\varepsilon} \odot v\left(\mathbf{V}^{\varepsilon}\right] \mid \mathrm{d} \mathcal{H}^{2}(\mathbf{X})\right\}\right. \\
& \stackrel{\text { Hölder }}{\leqslant} \sqrt{2} \gamma|\Sigma|^{1 / 2} \varepsilon^{\frac{3}{2}}\left(\int_{\Sigma^{\varepsilon}}\left|\mathcal{E}\left(\mathbf{V}^{\varepsilon}\right)\right|^{2} \mathrm{~d} \mathbf{X}\right)^{1 / 2}+\gamma \varepsilon \int_{J_{\mathbf{V}^{\varepsilon}}} \mid\left[\mathbf{V}^{\varepsilon} \odot v\left(\mathbf{V}^{\varepsilon}\right] \mid \mathrm{d} \mathcal{H}^{2}(\mathbf{X})\right. \\
& \stackrel{\text { Young }}{\leqslant} \frac{\mu}{3} \int_{\Sigma^{\varepsilon}}\left|\mathcal{E}\left(\mathbf{V}^{\varepsilon}\right)\right|^{2} \mathrm{~d} \mathbf{X}+\gamma \varepsilon \int_{J_{\mathbf{V}^{\varepsilon}}}\left|\left[\mathbf{V}^{\varepsilon} \odot v\left(\mathbf{V}^{\varepsilon}\right)\right]\right| \mathrm{d} \mathcal{H}^{2}(\mathbf{X})+6 \frac{\gamma^{2}}{\mu}|\Sigma| \varepsilon^{3} .
\end{aligned}
$$

and (6.7) is proved. Then (6.8) follows from (6.1), (6.7) since Kirchhoff cinematic restriction entails (6.2), (6.3), that is to say $J_{\mathbf{v}^{\varepsilon}}$ is the union of ribbons of height $2 \varepsilon$.

\section{REFERENCES}

1. Acerbi, E., Buttazzo, G., \& Percivale, D. Thin inclusion in nonlinear elasticity. J. Reine Angew. Math. 386, (1988) 99-115.

2. Ambrosio, L. Existence theory for a new class of variational problems. Arch. Rational Mech. Anal. 111, (1990) 291-322.

3. Ambrosio, L., Coscia, A., \& Dal Maso, G. Fine properties of functions with bounded deformation. Arch. Rat. Mech. Anal. 139, (1997) 201-238.

4. Ambrosio, L. \& Braides, A. Energies in SBV and variational models in fracture mechanics. Homogeneization and Appl. to Material sciences 9, (Nice, 1995) GAKUTO Int. Ser. Math. Sci. Appl.

5. Anzellotti, G., Baldo, S., \& Percivale, D. Dimension reduction in variational problems, asymptotic development in $\Gamma$-convergence and thin structures in elasticity. Asymptotic Anal. 9, (1994) 61-100.

6. Baiocchi, C., Buttazzo, G., Gastaldi, F., \& Tomarelli, F. General existence theorems for unilateral problems in continuum mechanics. Arch. Rat. Mech. Anal. 100, (1988) 149-189.

7. Barenblatt, G. I. The formation of equilibrium cracks during brittle fracture, general ideas and hypotheses. Axially symmetric cracks. Appl. Math. Mech. (PMM) 23, (1959) 622-636.

8. Bhattacharya, K. \& Braides, A. Thin films with many small cracks. Prepr. SISSA 36, (1999) 1-16. 
9. Bellettini, G., Coscia, A., \& Dal Maso, G. Compactness and lower semi-continuity properties in $\operatorname{SBD}(\Omega)$. Math. Z. 228, (1998) 337-351.

10. Braides, A., Dal Maso, G., \& Garroni, A. Variational formulation of softening phenomena in fracture mechanics: the one-dimensional case. Arch. Rat. Mech. Anal. 146, (1999) 23-58.

11. BRAIDES, A. \& ChIAdò-PiAT, V. Integral representation results for functionals defined on $\operatorname{SBV}\left(\Omega, \mathbb{R}^{m}\right)$. J. Math. Pures Appl. 75, (1996) 595-626.

12. Braides, A. \& FonseCA, I. Brittle thin films. Appl. Math. Opt. 44, (2001) 299-323.

13. BRAides, A., FonsecA, I., \& FrANCFORT, G. 3D-2D asymptotic analysis for inhomogeneous thin films. Indiana Univ. Math. J. 49, (2000) 1367-1404.

14. Carriero, M., Leaci, A., \& Tomarelli, F. Plastic free discontinuities and special bounded hessian. C. R. Acad. Sci. Paris 314, (1992) 595-600.

15. Carriero, M., Leaci, A., \& Tomarelli, F. Special Bounded Hessian and elastic-plastic plate. Rend. Accad. Naz. delle Scienze (dei XL), (109) XV, (1992) 223-258.

16. Carriero, M., Leaci, A., \& Tomarelli, F. Strong solution for an Elastic Plastic Plate. Calc. Var. 2, (1994) 219-240.

17. Carriero, M., Leaci, A., \& Tomarelli, F. Free gradient discontinuities. In: Bouchitté, Buttazzo, \& SuQuet (eds), Calculus of Variations, Homogeneization and Continuum Mechanics. World Scientific, Singapore (1994) pp. 131-147.

18. Casarino, V. \& Percivale, D. A variational model for non linear elastic plates. J. Convex Anal. 3, (1996) 221-243.

19. Ciarlet, P. G. Mathematical Elasticity, vol II: Theory of Plates, Studies in Math. and its Appl.. NorthHolland, Amsterdam (1997).

20. Colombo, F. \& Tomarelli, F. Boundary value problems and obstacle problems for an elastic body with free cracks. to appear.

21. Dal Maso, G. An Introduction to Gamma Convergence. Birkhäuser, PNLDE 8, (1993).

22. De Giorgi, E. \& Franzoni, T. Su un tipo di convergenza variazionale. Atti Accad. Naz. Lincei Rend. Cl. Sci. Mat. 58, (1975) 842-850.

23. De Giorgi, E. $\Gamma$-convergenza e G-convergenza. Boll. Un. Mat. Ital. 5 14-A, (1977) 213-220.

24. De Giorgi, E. Free discontinuity problems in calculus of variations. In: DaUtRAY, R. (ed), Frontiers in Pure \& Applied Mathematics. pp. 55-61. North-Holland, Amsterdam (1991).

25. De Giorgi, E. \& Ambrosio, L. Un nuovo tipo di funzionale del Calcolo delle Variazioni. Atti Accad. Naz. Lincei, Rend. Cl. Sci. Fis. Mat. Natur. 82, (1988) 199-210.

26. Demengel, F. Fonctions a hessien borné. Ann. Inst. Fourier 34, (1984) 155-190.

27. Del Piero, G. One-dimensional ductile-brittle transition, yielding and structured deformations. Prepr. Dip. Ing. Univ. Ferrara 42, (1999).

28. Del Piero, G. \& Owen, D. R. Structured deformations of continua. Arch. Rat. Mech. Anal. 124, (1993) 99-155.

29. Del Piero, G. \& Trushininovsky, L. A one-dimensional model for localized and distributed failure. J. Phys., IV France, pr. 8, (1998) 95-102.

30. Federer, H. Geometric Measure Theory. Springer, Berlin (1969).

31. Fonseca, I. \& Francfort, G. 3D-2D asymptotic analysis of optimal design problem for thin films. J. reine angew. Math. 505, (1998) 173-202.

32. Griffith, A. A. The phenomenon of rupture and flow in solids. Phyl. Trans. Roy. Soc. A 221, (1920) 163-198.

33. KIRChHOFF, G. Über das Gleichgewicht und die Bewegung einer elastischen Scheibe. J. Reine Angew. Math 40, (1850) 51-88.

34. Landau, L. D. \& Lifshitz, E. M. Theory of Elasticity, 7 Th. Phys. Pergamon Press, (1959).

35. Lions, J. L. Problèmes aux limites en théorie des distributions. Acta Math. 94, (1955) 13-153.

36. Percivale, D. Perfectly Plastic Plates: a variational definition. J. Reine Angew. Math. 411, (1990) 3950. 
37. Percivale, D. \& Tomarelli, F. Scaled Korn-Poincaré inequality in BD and a model of elastic plastic cantilever. Asymptotic Analysis 23, (2000) 291-311.

38. Percivale, D. \& Tomarelli, F. From Special Bounded Deformation to Special Bounded Hessian: the elastic-plastic linear beam, Quad. 496/P Dip. Mathematicq Politenico di Milano,(2002) 1-33.

39. Podio-Guidugli, P. Constraint and scaling methods to derive shell theory from three-dimensional elasticity. Riv. Math. Univ. Parma 16, (1990) 72-83.

40. Savarè, G. \& Tomarelli, F. Superposition and Chain Rule for Bounded Hessian Functions. Advances in Math. 140, (1998) 237-281.

41. Save, M. A. \& Massonet, C. E. Plastic Analysis and design of plates, shells and disks. North-Holland Ser. in Appl. Math. and Mech. (1972).

42. Temam, R. Problèmes Mathematiques en Plasticité. Gauthier-Vllars, Paris (1983).

43. Tomarelli, F. Special Bounded Hessian and partial regularity of equilibrium for a plastic plate. In: Buttazzo, G., Galdi, G. P. \& Zanghirati, L. (eds), Devel. in PDE and Applications to Mathematical Physics. pp. 235-240. Plenum Press, N.Y. (1992).

44. Temam, R. \& Strang, G. Functions of bounded deformation. Arch. Rat. Mech. Anal. 75, (1980) 7-21.

45. Truskinowsky, L. Fracture as a phase transition. In: BatzA, R. C. \& Beatty, M. F. (eds), Contemporary Research in the Mechanics and Mathematics of Materials (ded. to J. L. ERICKSEN). C.I.M.N.E., Barcelona (1996) pp. 322-332.

46. ZIEMER, W. P. Weakly Differentiable Functions. Springer, N.Y. (1988). 